\title{
Projeto de Controladores para Sistemas de Segunda Ordem com Atraso via Resposta em Frequência
}

\section{Nelson José Bonfim Dantas}

Orientador: Prof. Dr. Carlos Eduardo Trabuco Dórea

Co-orientador: Prof. Dr. José Mário Araújo

Dissertação de Mestrado apresentada ao Programa de Pós-Graduação em Engenharia Mecatrônica da UFRN como parte dos requisitos para obtenção do título de Mestre em Ciências.

Número de ordem PEM: M018

Natal, RN, maio de 2019 
Universidade Federal do Rio Grande do Norte - UFRN

Sistemas de Biblioteca - SISBI

Catalogação de Publicação na Fonte. UFRN - Biblioteca Central Zila Mamede

Dantas, Nelson José Bonfim.

Projeto de controladores para Sistemas de Segunda Ordem com Atraso via Resposta em Frequência / Nelson José Bonfim Dantas. - 2019.

58f.: il.

Dissertação (Mestrado) - Universidade Federal do Rio Grande do Norte, Centro de Tecnologia, Programa de Pós Graduação em Engenharia Mecatrônica, Natal, 2019.

Orientador: Dr. Carlos Eduardo Trabuco Dórea.

Co-orientador: Dr. José Mário Araújo

1. Sistemas de Segunda Ordem - Dissertação. 2. Sistemas com Atraso Dissertação. 3. Resposta em Frequência - Dissertação. I. Dórea, Carlos Eduardo Trabuco. II. Araújo, José Mário. III. Título. 


\section{Resumo}

A solução de problemas de controle para sistemas de segunda ordem com atraso constitui um dos desafios da engenharia. Fenômenos como vibrações mecânicas, ressonância e oscilações, podem ser descritos matematicamente por equações diferenciais de segunda ordem, nomeando estes sistemas como sistemas de segunda ordem. Este trabalho tem como objetivo principal projetar controladores para sistemas de segunda ordem com atraso, utilizando a abordagem por resposta em frequência, dispensando a necessidade de aproximações para o atraso, garantindo soluções estáveis sem necessidade de análise à posteriori. Definindo matematicamente o problema de controle como um problema de otimização, é feita uma busca pelos ganhos do controlador com a utilização de um algoritmo genético, tendo como parâmetro de projeto garantir que a curva de Nyquist do sistema permaneça a uma distância suficiente da região de instabilidade. Essa metodologia visa garantir que os ganhos calculados resultem em sistemas robustos para que mesmo sujeitos a alterações de parâmetros, a estabilidade seja garantida. Os resultados obtidos de simulações ao final do trabalho se mostraram satisfatórios dentro das especificações desejadas.

Palavras-chave: Sistemas de Segunda Ordem, Sistemas com Atraso, Matriz de Receptância, Resposta em Frequência, Algoritmo Genético. 



\section{Abstract}

The solution of second order systems control problems is located in the engineering challenges.Phenomena such as mechanical vibrations, resonance and so many can be described mathematically by these types of systems. This work has as main objective to solve a problem of design of controller by state feedback for second order systems with delay using frequency response approach, which dispenses with the need for approximations to the delay, thus ensuring that the solutions found do not require a posteriori analysis. By mathematically defining the control problem as an optimization problem, a search for controller gains is made using a genetic algorithm, with the project parameter to ensure that the Nyquist curve of the system remains at a sufficient distance from the instability region. This methodology aims to ensure that the calculated gains result in robust systems so that even subject to parameter changes, still remain stable. The results of simulations at the end of the work were satisfactory within the desired specifications.

Keywords: Second Order Systems, Delayed Systems, Receptance Matrix, Frequency Response, Genetic Algorithm. 



\section{Sumário}

Sumário

Lista de Figuras iii

$\begin{array}{ll}\text { Lista de Tabelas } & \text { v }\end{array}$

Lista de Símbolos e Abreviaturas vii

1 Introdução 1

2 Teoria 3

$2.1 \quad$ Sistemas de Segunda Ordem . . . . . . . . . . . . . . . . . 3

2.1.1 Representação de Primeira Ordem . . . . . . . . . . . . . . . . . . 4

2.1 .2 Problema de Autovalor Quadrático (QEP) . . . . . . . . . . . . . . . . . 5

2.1 .3 Realimentação de Estados . . . . . . . . . . . . . . . 5

2.1 .4 Realimentação Derivativa . . . . . . . . . . . . . . . 6

2.2 Alocação de Autovalores para Sistemas de Segunda Ordem . . . . . . . . 6

2.2.1 O Método da Receptância . . . . . . . . . . . . . . 7

$2.2 .2 \quad$ A Matriz de Receptância . . . . . . . . . . . . . . . . . . . . . . . 7

2.2 .3 Sistema com Atraso $\ldots \ldots \ldots$. . . . . . . . . . . . 9

2.3 Resposta em Frequência . . . . . . . . . . . . . . . . . . . . 10

2.3 .1 Resposta em Regime Permanente a Entradas Senoidais . . . . . . 10

$2.3 .2 \quad$ O Diagrama Polar, ou Diagrama de Nyquist . . . . . . . . . . . . . . 12

2.3 .3 Critério de Estabilidade de Nyquist . . . . . . . . . . . . 13

$\begin{array}{lll}3 & \text { Formulação do Problema } & 15\end{array}$

3.1 O Problema da Alocação Indesejada . . . . . . . . . . . . . . . . . . . . 16

3.2 Abordagem Usando o Preditor de Smith . . . . . . . . . . . . . . . . . . 17

$3.3 \quad$ A Ideia da Abordagem por Resposta em Frequência . . . . . . . . . . . . 18

3.4 Abordagem do Problema no Domínio da Frequência . . . . . . . . . . . . . 18

3.5 Parâmetro de Projeto e Formulação Matemática . . . . . . . . . . . . . . 19

3.6 Otimização de Desempenho $\ldots \ldots \ldots$. . . . . . . . . . . . . 20

4 Implementação do Método Proposto 23

4.1 Solução do Problema de Otimização . . . . . . . . . . . . . . . . . . 23

4.2 A Função Objetivo . . . . . . . . . . . . . . . . . . . . . . . . . . . . . . . . . . . . . . .

4.3 A Função de Restrições . . . . . . . . . . . . . . . . . . . . . . . . . 26 
\begin{tabular}{|lll}
\hline Experimentos e Resultados & 27
\end{tabular}

5.1 Exemplo 1. . . . . . . . . . . . . . . . . . . . . . . . . . . . .

5.2 Exemplo 2 . . . . . . . . . . . . . . . . . . . 30

5.3 Exemplo $3 \ldots \ldots \ldots \ldots \ldots \ldots$. . . . . . . . . . . . . . . . 37

5.4 Exemplo $4 \ldots \ldots \ldots \ldots \ldots \ldots$

6 Conclusão 41

Referências bibliográficas 42 


\section{Lista de Figuras}

$2.1 \quad$ Sistema Massa-Mola-Amortecedor . . . . . . . . . . . . . . . 3

$2.2 \quad$ Exemplo de Diagrama de Nyquist para Sistema modelado por $G(s)$. . . . 12

2.3 Diagrama de Nyquist com a Parte Espelhada . . . . . . . . . . . . 13

3.1 Sistema de Segunda Ordem com Atraso - 1 Grau de Liberdade . . . . . . 16

3.2 Curva da Equação Característica do Sistema da Figura $Q(s)]$. . . . . . . 17

3.3 Diagrama de Nyquist Exemplo de Circunferência $\mathrm{M}_{s} \ldots \ldots$. . . . . . . . . 19

3.4 Diagrama de Nyquist de um Sistema Genérico para Exemplo de um Caso de Instabilidade . . . . . . . . . . . . . . . . . . 21

5.1 Sistema massa-mola-amortecedor de um grau de liberdade . . . . . . . . 27

5.2 Diagrama de Nyquist Ganhos sem Restrição Adicional . . . . . . . . . . . . 28

5.3 Diagrama de Nyquist Ganhos com Restrição Adicional . . . . . . . . . . . 29

5.4 Resposta do Estado $\mathbf{x}(t)$ para condições iniciais $\dot{\mathbf{x}}(0)=0$ e $\mathbf{x}(0)=0,3$. . 29

$5.5 \quad$ Resposta do Estado $\dot{\mathbf{x}}(t)$ para condições iniciais $\dot{\mathbf{x}}(0)=0$ e $\mathbf{x}(0)=0,3$. . 30

5.6 Diagrama de Nyquist Exemplo 2 - Método Proposto SRA . . . . . . . . . 32

5.7 Diagrama de Nyquist Exemplo 2 - Alocação com Atraso . . . . . . . . . . 32

5.8 Resposta do estado $\mathbf{x}(t)$ para o Exemplo $2 \ldots \ldots . \ldots . . \ldots . . \ldots 33$

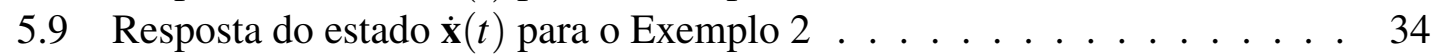

5.10 Diagrama de Nyquist Exemplo 2 alocação desconsiderando o atraso . . . 35

5.11 Diagrama de Nyquist Exemplo 2 - Controlador com restrição adicional . . 35

5.12 Resposta para os estados $\mathbf{x}(t)$ e $\dot{\mathbf{x}}(t)$ para os ganhos de controlador com restrição adicional . . . . . . . . . . . . . 36

5.13 Diagrama de Nyquist do Exemplo 3 para os ganhos obtidos pelo método proposto com a restrição adicional $\ldots \ldots \ldots 37$

5.14 Resposta Temporal Sistema 3 Graus de Liberdade - Exemplo 3 . . . . . . 38

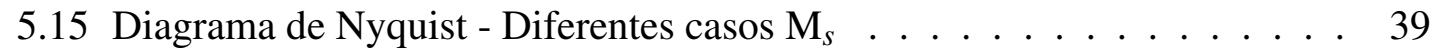

5.16 Resposta do Estado $x(t)$ - Diferentes casos $\mathrm{M}_{s} \ldots \ldots \ldots$. . . . . . . 40 



\section{Lista de Tabelas}

5.1 Tabela de Ganhos de Realimentação de Estado . . . . . . . . . . . . . . . 28

5.2 Margens de ganho . . . . . . . . . . . . . . . . 30

5.3 Tabela de Ganhos de Realimentação de Estado - Exemplo 2 . . . . . . . . 31

5.4 Margens de ganho - Exemplo 2 . . . . . . . . . . . . . . . 31

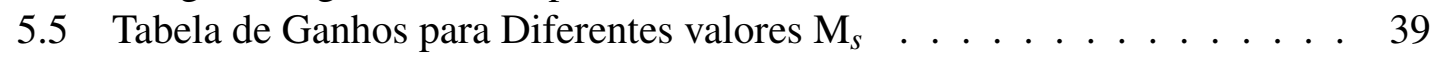





\section{Lista de Símbolos e Abreviaturas}

$\zeta \quad$ Coeficiente de amortecimento da resposta temporal, veja equação (5.0), página 30

AVC Active Vibration Control, veja equação (2.19), página 7

CRA Com Restrição Adicional, veja equação (5.0), página 29

GA Genetic Algorithm, veja equação (4.0), página 23

GM Gain Margin - Margem de Ganho, veja equação (5.0), página 30

PM Phase Margin - Margem de Fase, veja equação (5.0), página 30

QEP Quadratic Eingenvalue Problem, veja equação (2.8), página 5

SISO Single Input Single Output, veja equação (2.4), página 4

SPD Semiplano Direito, veja equação (2.37), página 14

SPE Semiplano Esquerdo, veja equação (2.36), página 13

SRA Sem Restrição Adicional, veja equação (5.0), página 29 



\section{Capítulo 1}

\section{Introdução}

Fenômenos físicos como a ressonância em sistemas vibratórios, oscilações em redes elétricas, vibro-acústica e vários outros, possuem em comum a abordagem matemática utilizada na representação de seus modelos (Balas 1982, Vandervelde 1986). Geralmente a descrição desses fenômenos é feita utilizando-se equações diferenciais de segunda ordem, o que classifica tais sistemas como sistemas de segunda ordem.

Os modelos matemáticos que representam esses sistemas podem ser escritos em sua forma original, como um sistema matricial de equações diferenciais de segunda ordem, onde as constantes e variáveis das equações tem uma relação física direta com o modelo real, ou traduzidos para um modelo de primeira ordem, onde a representação é tida apenas com equações diferenciais de primeira ordem. Em Datta (2004) algumas representações de primeira ordem para sistemas de segunda ordem são apresentadas, e algumas desvantagens com relação ao modelo original são discutidas.

A abordagem por representações na forma original conduz ao problema de autovalor quadrático, que consiste na determinação do conjunto de $2 n$ autovalores do sistema, e consequentemente, constituindo parte do problema de alocação de autovalores. Em Juang \& Maghami (1990) é apresentado um método para solução do problema de alocação de autovalores, baseado em decomposição por valor singular. Outras abordagens podem ser encontradas em Datta et al. (1997).

O estudo de sistemas de segunda ordem tem ganhado cada vez mais notoriedade na comunidade da engenharia devido a sua relevância prática, e tem apresentado soluções para vários desafios. Uma das dificuldades de se trabalhar com os modelos matemáticos obtidos pelas equações diferenciais de segunda ordem está na definição dos elementos das matrizes que caracterizam o sistema (rigidez, massa e elasticidade). O conceito de matriz de receptância, apresentado em Mottershead \& Ram (2007), que permite que os modelos dos sistema sejam obtidos experimentalmente, é utilizado em vários trabalhos relacionados (Araújo 2018b, Araújo \& Santos 2018, Liu \& Yuan 2016, Santos et al. 2018). O método da receptância se mostra muito vantajoso do ponto de vista da precisão no controle de sistemas com grande número de graus de liberdade e complexa estrutura de seus elementos (Mottershead \& Ram 2007).

Quando existe atraso de transporte nos modelos físicos, infinitos autovalores podem ser admitidos para o sistema, mas somente um conjunto de $2 n$ destes tem influência no comportamento dinâmico da resposta. A ideia de alocar apenas um conjunto de autova- 
lores dentre todos existentes constitui o problema de alocação parcial (Ram et al. 2011, Singh et al. 2014, Araújo 2018b).

A distinção entre os autovalores que influenciam ou não, no comportamento do sistema, não é trivial, e faz indispensável uma análise à posteriori dos valores de ganhos encontrados como solução para o chamado problema de alocação parcial de autovalores. Essa dificuldade de distinguir entre os autovalores que atuam diretamente na dinâmica da resposta, chamados autovalores primários, e os que não atuam, chamados autovalores secundários, é ilustrada em Ram et al. (2009), e a solução proposta pelo autor passa pela utilização de aproximações por séries de Taylor para o termo exponencial, obtendo resultados mais precisos à medida que se tenham aproximações de ordem mais elevada, o que todavia aumenta a complexidade de projeto. Em Araújo (2018a) a aproximação de Padé é usada como alternativa a aproximação polinomial de Taylor usada em Ram et al. (2009).

Motivado pela possibilidade de levantamento de modelos de sistemas de segunda ordem de maneira experimental, justificado pela dispensa da necessidade de aproximações para o atraso, este trabalho tem como objetivo principal propor a solução de um problema de controle por realimentação de estado para um sistema de segunda ordem com atraso, para tanto, uma abordagem por resposta em frequência é adotada. Definindo matematicamente o problema de controle como um problema de otimização dos ganhos do controlador, utiliza-se como ferramenta de solução o algoritmo genético, objetivando atender especificações de projeto que garanta solução robusta, para que mesmo sujeito a alterações de parâmetros o sistema permaneça estável.

O restante do trabalho está disposto da seguinte maneira:

- Capítulo2 Apresenta a teoria matemática básica para a compreensão do problema abordado e desenvolvimento do trabalho.

- Capítulo 3 . Apresenta o problema com equacionamento matemático e detalhes importantes sobre as vantagens do método a ser utilizado.

- Capítulo 4. Apresenta o método desenvolvido para solução do problema apresentado no Capítulo 3.

- Capítulo 5. Apresenta os resultados obtidos com a utilização do método proposto, através de exemplos numéricos emprestados de outros trabalhos com objetivo de obter parâmetros de comparação dos resultados alcançados.

- Capítulo 6. Traz comentários conclusivos e sugestões para futuros trabalhos relacionados. 


\section{Capítulo 2}

\section{Teoria}

\subsection{Sistemas de Segunda Ordem}

Equações diferenciais de segunda ordem descrevem o comportamento dinâmico de diversos tipos de sistemas, como por exemplo, sistemas mecânicos vibratórios. Estes tipos de sistemas são classificados como sistemas de segunda ordem e são descritos por um número $n$ destas equações diferenciais, uma para cada grau de liberdade do sistema. A importância destes modelos é evidenciada em estudos e publicações recentes no segmento como em Araújo (2018b) e Santos et al. (2018).

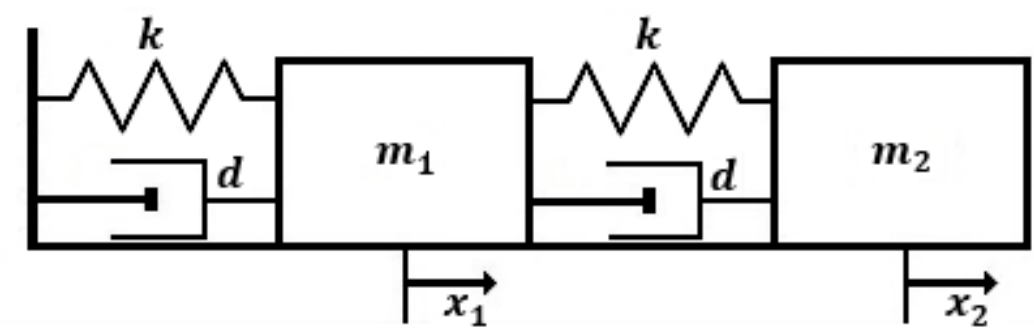

Figura 2.1: Sistema Massa-Mola-Amortecedor

O sistema mostrado na Figura 2.1 pode ser modelado por equações diferenciais, segundo as leis de Newton da mecânica clássica, da seguinte forma:

$$
\begin{aligned}
m_{1} \ddot{x}_{1}(t)+d\left(2 \dot{x}_{1}(t)-\dot{x}_{2}(t)\right)+k\left(2 x_{1}(t)-x_{2}(t)\right) & =0 \\
m_{2} \ddot{x}_{2}(t)+d\left(\dot{x}_{2}(t)-\dot{x}_{1}(t)\right)+k\left(x_{2}(t)-x_{1}(t)\right) & =u
\end{aligned}
$$

onde os coeficientes $m, d$ e $k$, indicam respectivamente, as massas, amortecimento e rigidez de cada componente indicado na figura. Os deslocamentos são apontados pelo estado $x$, e suas derivadas, $\dot{x}$ e $\ddot{x}$ indicam velocidade e aceleração.

Em forma matricial as equações (2.1) e 2.2) podem ser reescritas da seguinte maneira:

$$
\left[\begin{array}{cc}
m_{1} & 0 \\
0 & m_{2}
\end{array}\right]\left[\begin{array}{c}
\ddot{x}_{1} \\
\ddot{x}_{2}
\end{array}\right]+\left[\begin{array}{cc}
2 d & -d \\
-d & d
\end{array}\right]\left[\begin{array}{c}
\dot{x}_{1} \\
\dot{x}_{2}
\end{array}\right]+\left[\begin{array}{cc}
2 k & -k \\
-k & k
\end{array}\right]\left[\begin{array}{l}
x_{1} \\
x_{2}
\end{array}\right]=\left[\begin{array}{l}
0 \\
1
\end{array}\right] u
$$


que de forma abreviada fica como:

$$
\mathbf{M} \ddot{\mathbf{x}}+\mathbf{D} \dot{\mathbf{x}}+\mathbf{K} \mathbf{x}=\mathbf{B u}
$$

com

$$
\mathbf{M}=\left[\begin{array}{cc}
m_{1} & 0 \\
0 & m_{2}
\end{array}\right], \mathbf{D}=\left[\begin{array}{cc}
2 d & -d \\
-d & d
\end{array}\right], \mathbf{K}=\left[\begin{array}{cc}
2 k & -k \\
-k & k
\end{array}\right] e \mathbf{B}=\left[\begin{array}{l}
0 \\
1
\end{array}\right]
$$

Onde $\mathbf{M} \in \mathfrak{R}^{n \times n}$, é uma matriz de massas, na maioria dos casos definida positiva; $\mathbf{D} \in \mathfrak{R}^{n \times n}$ é uma matriz de amortecimento, geralmente, pelo menos, semidefinida positiva; $\mathbf{K} \in \mathfrak{R}^{n \times n}$ é uma matriz de rigidez, também sendo semidefinida positiva na maior parte das vezes; $\mathbf{B} \in \Re^{n \times m}$ é uma matriz de controle (também vista como matriz de influência ou de atuadores) de posto completo; $\mathbf{x} \in \mathfrak{R}^{n}$ é o vetor de deslocamento e $\mathbf{u} \in \mathfrak{R}^{m}$ é um vetor de entradas.

\subsubsection{Representação de Primeira Ordem}

A equação (2.4) representa um sistema de segunda ordem em sua forma original. Uma outra maneira de interpretar o sistema descrito em (2.4) é escrever o modelo por uma representação de primeira ordem, como por exemplo, no espaço de estados tradicional $\dot{\mathbf{x}}=\mathbf{A} \mathbf{x}+\mathbf{B} u$.

Definindo $\mathbf{x}=\tilde{\mathbf{x}}_{1}$ e $\dot{\mathbf{x}}=\tilde{\mathbf{x}}_{2}$, podemos escrever o sistema de segunda ordem da equação (2.4) como:

$$
\begin{aligned}
\dot{\tilde{\mathbf{x}}}_{1} & =\tilde{\mathbf{x}}_{2} \\
\dot{\tilde{\mathbf{x}}}_{2} & =-\mathbf{M}^{-1} \mathbf{K} \tilde{\mathbf{x}}_{1}-\mathbf{M}^{-1} \mathbf{D} \tilde{\mathbf{x}}_{2}+\mathbf{M}^{-1} \mathbf{B} u
\end{aligned}
$$

e em forma de matrizes

$$
\dot{\tilde{\mathbf{x}}}=\left[\begin{array}{c}
\dot{\mathbf{x}}_{1} \\
\tilde{\mathbf{x}}_{2}
\end{array}\right]=\left[\begin{array}{cc}
\mathbf{0} & \mathbf{I} \\
-\mathbf{M}^{-1} \mathbf{K} & -\mathbf{M}^{-1} \mathbf{D}
\end{array}\right]\left[\begin{array}{c}
\tilde{\mathbf{x}}_{1} \\
\tilde{\mathbf{x}}_{2}
\end{array}\right]+\left[\begin{array}{c}
\mathbf{0} \\
\mathbf{M}^{-1} \mathbf{B}
\end{array}\right] u
$$

ou de maneira abreviada

$$
\dot{\tilde{\mathbf{x}}}=\mathbf{A} \tilde{\mathbf{x}}+\mathbf{B} u
$$

com

$$
\mathbf{A}=\left[\begin{array}{cc}
\mathbf{0} & \mathbf{I} \\
-\mathbf{M}^{-1} \mathbf{K} & -\mathbf{M}^{-1} \mathbf{D}
\end{array}\right] \text { e } \mathbf{B}=\left[\begin{array}{c}
\mathbf{0} \\
\mathbf{M}^{-1} \mathbf{B}
\end{array}\right]
$$

Em Datta (2004) esta representação é denominada de representação de primeira ordem padrão, visto que outras formas de representação de primeira ordem para sistemas de segunda ordem são generalizadas pelo autor, como a forma de sistema descritor, por exemplo. Na literatura de controle, como em Ogata (2009) e Franklin et al. (2013), essa representação é vista como a representação de um sistema de equações diferenciais no espaço de estados, como é bem conhecida na engenharia. A motivação para se obter um modelo de primeira ordem está no fato de que as equações diferenciais de ordem um possuem solução mais simples do que as de ordem superior. Entretanto, algumas dificuldades 
numéricas aparecem no tratamento de sistemas representados desta forma, a começar pela duplicação do número de equações do sistema, e da análise da equação (2.7), fica evidente que para obtenção da matriz $\mathbf{A}$, a matriz $\mathbf{M}$ precisa ser inversível, e se a matriz $\mathbf{M}$ não for bem condicionada, sua inversa, e consequentemente a matriz $\mathbf{A}$, não serão computadas com precisão. Outro fato é que importantes propriedades exploráveis das matrizes de coeficientes $\mathbf{M}, \mathbf{D}$ e $\mathbf{K}$, como simetria e esparsidade, que são valiosas em problemas de grandes dimensões, são completamente perdidas quando se passa a representar um sistema de segunda ordem na forma da equação (2.7).

\subsubsection{Problema de Autovalor Quadrático (QEP)}

Este trabalho aborda sistemas de segunda ordem modelados em sua forma original, como na equação (2.4). Ao se trabalhar desta maneira, surge o problema de autovalor quadrático $(\mathrm{QEP})$ para solução homogênea $(u=0)$ da representação de (2.4) que pode ser vista fazendo $\mathbf{x}=\mathbf{z} e^{\lambda t}$, onde $\mathbf{z} \neq 0$ é um vetor constante, solução de:

$$
\mathbf{M} \ddot{\mathbf{x}}+\mathbf{D} \dot{\mathbf{x}}+\mathbf{K x}=\mathbf{0}
$$

chegando a:

$$
\left(\lambda^{2} \mathbf{M}+\lambda \mathbf{D}+\mathbf{K}\right) \mathbf{z} e^{\lambda t}=\mathbf{0}
$$

onde

$$
\left(\lambda^{2} \mathbf{M}+\lambda \mathbf{D}+\mathbf{K}\right) \mathbf{z} \equiv P(\lambda) \mathbf{z}=0
$$

$P(\lambda)$ é chamado Feixe Quadrático ou Matriz Lambda; $\lambda$ é chamado autovalor do sistema e $\mathbf{z}$ autovetor à direita associado a $\lambda$. O QEP é solucionado encontrando-se os $2 n$ valores de $\lambda$ solução da Equação (2.12):

$$
\operatorname{det}\left(\lambda^{2} \mathbf{M}+\lambda \mathbf{D}+\mathbf{K}\right)=0
$$

onde $n$ é a dimensão da Matriz Lambda. Outros métodos para solução do QEP são encontrados em Datta (2004).

\subsubsection{Realimentação de Estados}

Sistemas de segunda ordem geralmente representam sistemas mecânicos como o da Figura 2.1. onde o vetor de variáveis de estado, $\mathbf{x}$, representa o deslocamento do sistema e o vetor de derivadas, $\dot{\mathbf{x}}$, a velocidade. A tradicional técnica de realimentação de estados consiste em aplicar a lei de controle definida pela equação (2.13), em que f, $\mathbf{g} \in \mathfrak{R}^{n \times m}$ são vetores de ganho de realimentação, ao sistema modelado pela equação (2.4), chegando a (2.14).

$$
\begin{gathered}
u(t)=\mathbf{f}^{T} \dot{\mathbf{x}}(t)+\mathbf{g}^{T} \mathbf{x}(t) \\
\mathbf{M} \ddot{x}(t)+\left(\mathbf{D}-\mathbf{B} \mathbf{f}^{T}\right) \dot{x}(t)+\left(\mathbf{K}-\mathbf{B g}^{T}\right) x(t)=0
\end{gathered}
$$

O sistema realimentado representado por 2.14 dá origem ao seguinte feixe quadrá- 
tico:

$$
P(\lambda)=\lambda^{2} \mathbf{M}+\lambda\left(\mathbf{D}-\mathbf{B f}^{T}\right)+\left(\mathbf{K}-\mathbf{B g}^{T}\right)
$$

dessa forma, o comportamento dinâmico do sistema pode ser modificado através de alterações nas matrizes de amortecimento e rigidez de (2.14).

De maneira análoga a (2.4), 2.15) também possui uma representação de primeira ordem que pode ser escrita na forma:

$$
\dot{\tilde{\mathbf{x}}}(t)=\left[\begin{array}{cc}
\mathbf{0} & \mathbf{I} \\
-\mathbf{M}^{-1}\left(\mathbf{K}-\mathbf{B g}^{T}\right) & -\mathbf{M}^{-1}\left(\mathbf{D}-\mathbf{B} \mathbf{f}^{T}\right)
\end{array}\right] \tilde{\mathbf{x}}(t)
$$

que pode ser vista na forma clássica de realimentação de estados $\dot{\tilde{\mathbf{x}}}(t)=(\tilde{\mathbf{A}}+\tilde{\mathbf{B}} \mathbf{H}) \tilde{\mathbf{x}}(t)$, com

$$
\tilde{\mathbf{A}}=\left[\begin{array}{cc}
0 & I \\
-\mathbf{M}^{-1} \mathbf{K} & -\mathbf{M}^{-1} \mathbf{D}
\end{array}\right], \tilde{\mathbf{B}}=\left[\begin{array}{c}
0 \\
\mathbf{M}^{-1} \mathbf{B}
\end{array}\right] \text { e } \mathbf{H}=\left[\begin{array}{ll}
\mathbf{g}^{T} & \mathbf{f}^{T}
\end{array}\right]
$$

\subsubsection{Realimentação Derivativa}

Em sistemas mecânicos, como os da Figura 2.1, os sinais da derivada dos estados são mais fáceis de serem obtidos do que o sinal dos estados. os dados referentes a deslocamento são mais difíceis de serem obtidos com precisão do que os dados de velocidade e aceleração (Zhang et al. 2014). Assim a realimentação por derivada de estados torna-se uma alternativa desejada na solução de problemas de controle nestes tipos de sistema. De maneira análoga a realimentação de estado, a realimentação derivativa é governada pela seguinte lei de controle:

$$
u(t)=\mathbf{f}_{d}^{T} \ddot{\mathbf{x}}(t)+\mathbf{g}_{d}^{T} \dot{\mathbf{x}}(t)
$$

em que $\mathbf{f}_{d}, \mathbf{g}_{d} \in \mathfrak{R}^{n \times m}$ são vetores de ganho de realimentação. Substituindo (2.18) em (2.4):

$$
\begin{array}{r}
\mathbf{M} \ddot{x}(t)+\mathbf{D} \dot{x}(t)+\mathbf{K} x(t)=\mathbf{B}\left(\mathbf{f}_{d}^{T} \ddot{x}(t)+\mathbf{g}_{d}^{T} \dot{x}(t)\right) \\
\left(\mathbf{M}-\mathbf{B} \mathbf{f}_{d}^{T}\right) \ddot{x}(t)+\left(\mathbf{D}-\mathbf{B g}_{d}^{T}\right) \dot{x}(t)+\mathbf{K} x(t)=0
\end{array}
$$

dando origem ao seguinte feixe quadrático

$$
\mathbf{P}_{\mathbf{d}}(\lambda)=\lambda^{2}\left(\mathbf{M}-\mathbf{B f}_{d}^{T}\right)+\lambda\left(\mathbf{D}-\mathbf{B g}_{d}^{T}\right)+\mathbf{K}
$$

nessa configuração, as matrizes a serem alteradas pela realimentação por derivada de estados são as matrizes de massa e de amortecimento, onde o subscrito " $d$ " relaciona a matriz a realimentação derivativa.

\subsection{Alocação de Autovalores para Sistemas de Segunda Ordem}

Por várias anos o problema de alocação de autovalores tem recebido atenção da comunidade de controle. Em Wonham (1967) foi mostrado que os polos de um sistema podem 


\subsection{ALOCAÇÃO DE AUTOVALORES PARA SISTEMAS DE SEGUNDA ORDEM 7}

ser alocados por realimentação de estados se o sistema for controlável. Em Chu \& Datta (1996) são apresentados métodos para solução do problema de alocação, definido como o problema de calcular vetores de ganho tal que o feixe quadrático de malha fechada (2.15) tenha um conjunto de autovalores e autovetores associados desejados. Em (Mottershead \& Ram 2007) é apresentado o método da receptância, utilizado no controle ativo de vibrações (AVC), mostrando-se vantajoso devido à possibilidade de se obter um modelo experimental do sistema, dispensando a necessidade de precisas definições dos elementos das matrizes M, D e K. Neste trabalho os sistemas de segunda ordem são descritos por matriz de receptância e o problema abordado é definido no Capítulo 3 .

\subsubsection{O Método da Receptância}

Em Ram et al. (2011) a alocação de autovalores é citada como sendo o inverso do QEP, uma vez que, dado um conjunto de autovalores desejados para o sistema, o problema passa a ser, encontrar um conjunto de ganhos de controlador ( $\mathbf{g}$ e f) que satisfaça o conjunto de autovalores desejados como solução do feixe quadrático dado pela equação (2.15). O problema de alocação de polos é solucionado em Mottershead \& Ram (2007), para o controle ativo de vibrações, usando um método baseado em medições, exaltando algumas vantagens em relação à abordagem convencional do espaço de estados, que utiliza as matrizes de massa, rigidez e amortecimento, obtidas pelo método dos elementos finitos. Por ser um método baseado unicamente em dados medidos de vibrações, não há necessidade de se determinar, ou conhecer, essas matrizes. A determinação das matrizes de rigidez (K) e de massas (M) não é tão desafiadora, visto que, segundo Mottershead \& Ram (2007), os elementos finitos destas matrizes podem ser facilmente determinadas com a utilização, por exemplo, de métodos variacionais. Entretanto, essa praticidade não se aplica a matriz de amortecimento (D), pois não existe uma abordagem semelhante que possa lidar com os diferentes tipos de amortecimento (viscoso, material, atrito, impacto, etc ...) presentes em estruturas físicas reais. Em alguns trabalhos que utilizam modelos no espaço de estados, os elementos da matriz de amortecimento são definidos sem seguir um critério físico solidamente estabelecido, ou adotando o amortecimento de Rayleigh. Em estudos convencionais de vibração levemente amortecida, esta métrica pode ser aceitável, mas em estudos de controle ativo o modelo de amortecimento é de extrema importância, por exemplo, na compreensão de estabilidade relativa (Vandervelde 1986).

\subsubsection{A Matriz de Receptância}

Considerando o controle de um sistema de segunda ordem por realimentação de estados definido por

$$
\begin{gathered}
\mathbf{M} \ddot{\mathbf{x}}(t)+\mathbf{D} \dot{\mathbf{x}}(t)+\mathbf{K} \mathbf{x}(t)=\mathbf{B} u(t) \\
u(t)=\mathbf{f}^{T} \dot{\mathbf{x}}(t)+\mathbf{g}^{T} \mathbf{x}(t)
\end{gathered}
$$

Onde $\mathbf{M}, \mathbf{D}, \mathbf{K} \in \mathfrak{R}^{n \times n} ; \mathbf{B}, \mathbf{f}, \mathbf{g} \in \mathfrak{R}^{n} ; \mathbf{x} \in \mathfrak{R}^{n}, \mathbf{M}=\mathbf{M}^{T}, \mathbf{D}=\mathbf{D}^{T}, \mathbf{K}=\mathbf{K}^{T} ; \mathbf{v}^{T} \mathbf{M v}>0$, $\mathbf{v}^{T} \mathbf{D v} \geq 0$ e $\mathbf{v}^{T} \mathbf{K v} \geq 0$ para qualquer $\mathbf{v} \neq \mathbf{0}, \mathbf{v} \in \Re^{n}$. 
Substituindo 2.21) em 2.20), assumindo $\mathbf{x}(t)=\mathbf{z} e^{s t}$, sendo $\mathbf{z}$ um vetor constante e $s$ a variável de Laplace, solução do sistema, tem-se:

$$
\begin{array}{r}
\left(\mathbf{M} s^{2}+\mathbf{D} s+\mathbf{K}-\mathbf{B}(\mathbf{g}+s \mathbf{f})^{T}\right) \mathbf{z}=\mathbf{0} \\
\left(\mathbf{M} s^{2}+\left(\mathbf{D}-\mathbf{B f}^{T}\right) s+\mathbf{K}-\mathbf{B g}^{T}\right) \mathbf{z}=\mathbf{0}
\end{array}
$$

Onde pode-se perceber que as componentes de malha fechada de rigidez e amortecimento são modificadas pela matriz de posto- $1, \mathbf{B}(\mathbf{g}+s \mathbf{f})^{T}$, consequência da realimentação de estados.

\section{A Fórmula de Sherman-Morrison}

A fórmula de Sherman-Morrison fornece a inversa de uma matriz com modificação de posto-1 em termos da inversa da matriz original, e como visto em Van Loan \& Golub (1983) e Golub \& Van Loan (2012) ela estabelece que, para uma dada matriz $\mathbf{A} \in \Re^{n \times n}$ quadrada e inversível e vetores colunas $\mathbf{u}$ e $\mathbf{v} \in \mathfrak{R}^{n}$ :

$$
\left(\mathbf{A}+\mathbf{u v}^{T}\right)^{-1}=\mathbf{A}^{-1}-\frac{\mathbf{A}^{-1} \mathbf{u v} \mathbf{v}^{T} \mathbf{A}^{-1}}{1+\mathbf{v}^{T} \mathbf{A}^{-1} \mathbf{u}}
$$

Aplicando a fórmula de Sherman-Morrison em (2.22) $\operatorname{com} \mathbf{A}=\left(\mathbf{M} s^{2}+\mathbf{D} s+\mathbf{K}\right), \mathbf{u}=$ $\mathbf{B}$ e $\mathbf{v}=-(\mathbf{g}+s \mathbf{f})$, temos:

$$
\hat{\mathbf{H}}(s)=\mathbf{H}(s)+\frac{\mathbf{H}(s) \mathbf{B}(\mathbf{g}+s \mathbf{f})^{T} \mathbf{H}(s)}{1-(\mathbf{g}+s \mathbf{f})^{T} \mathbf{H}(s) \mathbf{B}}
$$

onde $\hat{\mathbf{H}}(s)=\left(\mathbf{M} s^{2}+\mathbf{D} s+\mathbf{K}-\mathbf{B}(\mathbf{g}+s \mathbf{f})^{T}\right)^{-1}$ é definida em (Ram et al. 2009) como matriz de receptância de malha fechada e $\mathbf{H}(s)=\left(\mathbf{M} s^{2}+\mathbf{D} s+\mathbf{K}\right)^{-1}$ como matriz de receptância de malha aberta, que na prática pode ser medida pela resposta em frequência $\mathbf{H}(j \omega)$.

O problema de alocação de autovalores para o sistema em malha fechada representado na forma da equação 2.24, consiste em calcular os vetores de ganho $\mathbf{f}$ e $\mathbf{g}$ dado os autovalores desejados. A equação característica de (2.24) é definida como:

$$
1-(\mathbf{g}+s \mathbf{f})^{T} \mathbf{H}(s) \mathbf{B}=0
$$

e em Mottershead \& Ram (2007) a equação (2.25) é resolvida sendo reescrita da seguinte forma:

$$
\begin{gathered}
\left(\mathbf{g}+s_{k} \mathbf{f}\right)^{T} \mathbf{H}\left(s_{k}\right) \mathbf{B}=1 \\
\mathbf{r}_{k}^{T}\left(\mathbf{g}+s_{k} \mathbf{f}\right)=1, \quad k=1,2, \ldots, 2 n
\end{gathered}
$$

ou

$$
\mathbf{r}_{k}^{T} \mathbf{g}+s_{k} \mathbf{r}_{k}^{T} \mathbf{f}=1, \quad k=1,2, \ldots, 2 n
$$

com $\mathbf{r}_{k}=\mathbf{H}\left(s_{k}\right) \mathbf{B}$, onde o subescrito $k$ representa o $k$-ésimo autovalor, do conjunto de 
autovalores desejados. E na forma matricial:

$$
\left[\begin{array}{cc}
s_{1} \mathbf{r}_{1}^{T} & \mathbf{r}_{1}^{T} \\
s_{2} \mathbf{r}_{2}^{T} & \mathbf{r}_{2}^{T} \\
\vdots & \vdots \\
s_{2 n} \mathbf{r}_{2 n}^{T} & \mathbf{r}_{2 n}^{T}
\end{array}\right]\left[\begin{array}{c}
\mathbf{f} \\
\mathbf{g}
\end{array}\right]=\left[\begin{array}{c}
1 \\
1 \\
\vdots \\
1
\end{array}\right]
$$

e desta forma a solução, para $\mathbf{f}$ e $\mathbf{g}$, do problema de alocação de autovalor pode ser facilmente obtida com a inversão da matriz

$$
\mathbf{G}=\left[\begin{array}{cc}
s_{1} \mathbf{r}_{1}^{T} & \mathbf{r}_{1}^{T} \\
s_{2} \mathbf{r}_{2}^{T} & \mathbf{r}_{2}^{T} \\
\vdots & \vdots \\
s_{2 n} \mathbf{r}_{2 n}^{T} & \mathbf{r}_{2 n}^{T}
\end{array}\right]
$$

Teoremas que relacionam a inversibilidade da matriz $\mathbf{G}$ e a controlabilidade do sistema, dentre outros, são ricamente discutidos em Mottershead \& Ram (2007) e Mottershead \& Ram (2006).

\subsubsection{Sistema com Atraso}

Quando existe um atraso no tempo entre o estado medido e a ação de controle do atuador podemos representar a lei de ação de controle da seguinte maneira:

$$
u(t-\tau)=\mathbf{f}^{T} \dot{\mathbf{x}}(t-\tau)+\mathbf{g}^{T} \mathbf{x}(t-\tau)
$$

onde novamente $\mathbf{f}, \mathbf{g} \in \mathfrak{R}^{n}$ vetores de ganho do sistema, e desta forma, um sistema de controle de segunda ordem, como o da Figura 2.1. descrito pelo sistema de equações (2.4), com matrizes de massa $\mathbf{M} \in \mathfrak{R}^{n \times n}$, amortecimento $\mathbf{D} \in \mathfrak{R}^{n}$ e elasticidade $\mathbf{K} \in \mathfrak{R}^{n}$ pode ser escrito como:

$$
\mathbf{M} \ddot{\mathbf{x}}(t)+\mathbf{D} \dot{\mathbf{x}}(t)+\mathbf{K x}(t)=\mathbf{B} u(t-\tau)
$$

Aplicando-se os procedimentos da seção anterior, para obtenção da matriz de receptância do sistema de segunda ordem com atraso descrito pela equação $(2.28), \operatorname{com} \mathbf{x}(t)=\mathbf{z} e^{s t}$, sendo $\mathbf{z}$ um vetor constante e $s$ a variável de Laplace, solução do sistema, tem-se:

$$
\begin{gathered}
\left(\mathbf{M} s^{2}+\mathbf{D} s+\mathbf{K}-\mathbf{B}(\mathbf{g}+s \mathbf{f})^{T} e^{-s \tau}\right) \mathbf{z}=\mathbf{0} \\
\left(\mathbf{M} s^{2}+\left(\mathbf{D}-\mathbf{B f}^{T} e^{-s \tau}\right) s+\mathbf{K}-\mathbf{B g}^{T} e^{-s \tau}\right) \mathbf{z}=\mathbf{0}
\end{gathered}
$$

e aplicando-se a fórmula de Sherman-Morrison como em (2.22)

$$
\hat{\mathbf{H}}(s)=\mathbf{H}(s)+\frac{\mathbf{H}(s) \mathbf{B}(\mathbf{g}+s \mathbf{f})^{T} \mathbf{H}(s) e^{-s \tau}}{1-(\mathbf{g}+s \mathbf{f})^{T} \mathbf{H}(s) \mathbf{B} e^{-s \tau}}
$$


onde novamente, $\hat{\mathbf{H}}(s)=\left(\mathbf{M} s^{2}+\mathbf{D} s+\mathbf{K}-\mathbf{B}(\mathbf{g}+s \mathbf{f})^{T} e^{-s \tau}\right)^{-1}$ é definida como matriz de receptância de malha fechada e $\mathbf{H}(s)=\left(\mathbf{M} s^{2}+\mathbf{D} s+\mathbf{K}\right)^{-1}$ como matriz de receptância de malha aberta. A presença do atraso na equação (2.30) não modifica a possibilidade da obtenção de $\mathbf{H}(s)$ por sua resposta em frequência, uma das motivações para o desenvolvimento deste trabalho.

A equação característica da matriz de receptância de (2.30) é dada por:

$$
\begin{gathered}
1-(\mathbf{g}+s \mathbf{f})^{T} \mathbf{H}(s) \mathbf{B} e^{-s \tau}=0 \\
(\mathbf{g}+s \mathbf{f})^{T} \mathbf{H}(s) \mathbf{B}=e^{s \tau}
\end{gathered}
$$

e reescrevendo matricialmente

$$
\left[\begin{array}{cc}
s_{1} \mathbf{r}_{1}^{T} & \mathbf{r}_{1}^{T} \\
s_{2} \mathbf{r}_{2}^{T} & \mathbf{r}_{2}^{T} \\
\vdots & \vdots \\
s_{2 n} \mathbf{r}_{2 n}^{T} & \mathbf{r}_{2 n}^{T}
\end{array}\right]\left[\begin{array}{l}
\mathbf{f} \\
\mathbf{g}
\end{array}\right]=\left[\begin{array}{c}
e^{s_{1} \tau} \\
e^{s_{2} \tau} \\
\vdots \\
e^{s_{2 n} \tau}
\end{array}\right]
$$

com $\mathbf{r}_{k}=\mathbf{H}\left(s_{k}\right) \mathbf{B}$, onde o subscrito $k \leq 2 n$ representa o $k$-ésimo autovalor de malha fechada desejado do sistema de segunda ordem representado por (2.30).

Para um sistema sem atraso, como modelado por (2.24), o sistema de equações (2.26) possui um conjunto finito de soluções, controlando o comportamento dinâmico do sistema. Para o caso com atraso, descrito por (2.30), o conjunto de $2 n$ equações de (2.32) possui um conjunto infinito de soluções (devido a natureza exponencial do atraso), e não se pode garantir o controle da dinâmica do sistema. Na maioria dos casos a solução para lidar com o atraso passa pela realização de uma aproximação do termo exponencial, $e^{-s \tau}$, medida dispensável quando se trabalha com resposta em frequência.

\subsection{Resposta em Frequência}

Na literatura clássica de controle, como em Ogata (2009), o termo resposta em frequência é definido como a resposta em regime permanente de um sistema a uma entrada senoidal, o que significa experimentalmente, excitar um sistema com uma senoide na entrada e observar o comportamento da saída. Os trabalhos realizados por resposta em frequência geralmente são motivados pela bom desempenho ao lidar com incertezas no modelo da planta, facilidade com que informações experimentais podem ser usadas para fins de projeto e ações com a presença do atraso em transporte (Franklin et al. 2013).

\subsubsection{Resposta em Regime Permanente a Entradas Senoidais}

Considere o sistema linear, invariante no tempo descrito pela função de transferência racional:

$$
G(s)=\frac{Y(s)}{U(s)}=\frac{b_{m}\left(s-z_{1}\right)\left(s-z_{2}\right) \ldots\left(s-z_{m}\right)}{\left(s-p_{1}\right)\left(s-p_{2}\right) \ldots\left(s-p_{n}\right)}
$$


onde $U(s)$ e $Y(s)$ são as transformadas de Laplace dos sinais de entrada $u(t)$ e saída $y(t)$, respectivamente. Sendo $G(s)$ uma fração polinomial com polinômio de grau $m$ no numerador e um polinômio de grau $n$ no denominador, onde $n \geq m$. As $n$ raízes $p_{i}$, $i=1,2, \ldots, n$, do polinômio característico do denominador de $G(s)$, são os polos de $G(s)$, as $m$ raízes $z_{j}, j=1,2, \ldots, m$ do numerador de $G(s)$ são os zeros e $b_{m}$ uma constante arbitrária.

Para a seguinte entrada senoidal $u(t)=\operatorname{sen}(\omega t)$, cuja transformada de Laplace é dada por:

$$
U(s)=\frac{\omega}{s^{2}+\omega^{2}}=\frac{\omega}{(s-j \omega)(s+j \omega)}
$$

a transformada de Laplace da saída é dada por:

$$
Y(s)=G(s) U(s)=\frac{b_{m}\left(s-z_{1}\right)\left(s-z_{2}\right) \ldots\left(s-z_{m}\right)}{\left(s-p_{1}\right)\left(s-p_{2}\right) \ldots\left(s-p_{n}\right)} \frac{\omega}{(s-j \omega)(s+j \omega)}
$$

e assumindo $G(s)$ estável, para o critério de estabilidade BIBO, e com todos os polos distintos, por expansão em frações parciais de $Y(s)$ chega-se em:

$$
Y(s)=\frac{C_{1}}{s-p_{1}}+\frac{C_{2}}{s-p_{2}}+\cdots+\frac{C_{n}}{s-p_{n}}+\frac{C_{0}}{s-j \omega}+\frac{\bar{C}_{0}}{s+j \omega}
$$

e a resposta no domínio do tempo da saída, $y(t)$, transformada inversa de Laplace de $Y(s)$ é dada por:

$$
y(t)=C_{1} e^{p_{1} t}+C_{2} e^{p_{2} t}+\cdots+C_{n} e^{p_{n} t}+C_{0} e^{j \omega t}+\bar{C}_{0} e^{-j \omega t}
$$

e com $G(s)$ estável, os termos $e^{p_{i} t}$ tendem a zero, quando $t \rightarrow \infty$. Dessa forma, a resposta em regime permanente (ou estado estacionário) à entrada senoidal é dada por:

$$
y_{s S}(t)=C_{0} e^{j \omega t}+\bar{C}_{0} e^{-j \omega t}
$$

Os termos da resposta $C_{0}$ e $\bar{C}_{0}$ podem ser calculados como:

$$
\begin{gathered}
C_{0}=\left.Y(s)(s-j \omega)\right|_{s=j \omega}=\left.G(s) \frac{\omega}{(s+j \omega)}\right|_{s=j \omega}=\frac{G(j \omega)}{j 2}=\frac{|G(j \omega)|}{j 2} e^{j L G(j \omega)} \\
\bar{C}_{0}=\left.Y(s)(s+j \omega)\right|_{s=-j \omega}=\left.G(s) \frac{\omega}{(s-j \omega)}\right|_{s=-j \omega}=\frac{G(-j \omega)}{-j 2}=\frac{|G(j \omega)|}{-j 2} e^{-j L G(j \omega)}
\end{gathered}
$$

e a resposta transitória

$$
\begin{aligned}
y_{s s}(t) & =\frac{|G(j \omega)| e^{j L G(j \omega)}}{j 2} e^{j \omega t}+\frac{|G(j \omega)| e^{-j L G(j \omega)}}{-j 2} e^{-j \omega t} \\
& =|G(j \omega)|\left[\frac{e^{j(\omega t+\angle G(j \omega))}-e^{-j(\omega t+\angle G(j \omega))}}{j 2}\right]
\end{aligned}
$$


que, por fim, se resume a:

$$
y_{s s}(t)=|G(j \omega)| \operatorname{sen}(\omega t+\angle G(j \omega))
$$

uma senoide de mesma frequência $\omega$ da entrada, modificada em amplitude por $|G(j \omega)|$ e defasada de $\angle G(j \omega)$, o que se permite deduzir que a resposta em frequência de um sistema modelado por uma função de transferência $G(s)$ pode ser obtido pela relação de módulo e fase entre os sinais de entrada e saída, ou de maneira direta:

$$
\frac{Y(j \omega)}{X(j \omega)}=G(j \omega)
$$

A função $G(j \omega)$ é chamada na literatura de função de transferência senoidal, sendo uma grandeza complexa que pode ser representada pelo módulo e ângulo de fase, tendo como parâmetro a frequência, sendo mais comumente representada graficamente por diagrama de Bode ou gráfico logarítmico, diagrama de Nyquist ou polar ou ainda pelo diagrama do logaritmo do módulo versus ângulo de fase, a chamada carta de Nichols.

\subsubsection{O Diagrama Polar, ou Diagrama de Nyquist}

A representação gráfica escolhida para o desenvolvimento deste trabalho foi o diagrama de Nyquist, também chamado de diagrama polar. O diagrama polar de uma função de transferência senoidal $G(j \omega)$ é um gráfico do módulo de $G(j \omega)$ versus o ângulo de fase de $G(j \omega)$ em coordenadas polares, com $\omega$ variando de zero a infinito (Ogata 2009). Um exemplo de diagrama de Nyquist de um sistema de segunda ordem típico dado pela equação (2.34) é mostrado na Figura 2.2.

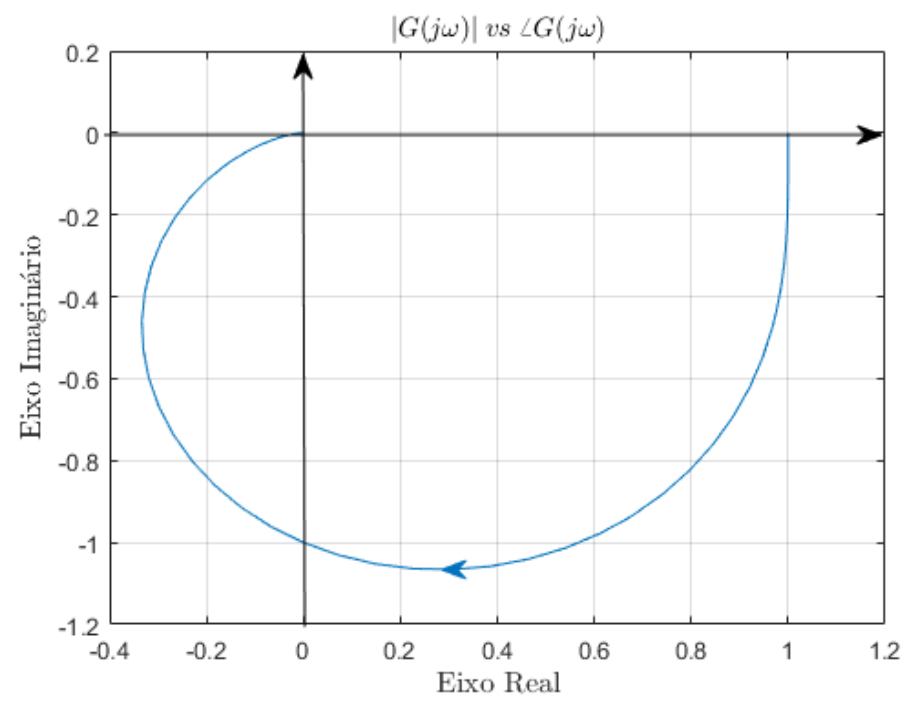

Figura 2.2: Exemplo de Diagrama de Nyquist para Sistema modelado por $G(s)$

$$
G(s)=\frac{1}{s^{2}+s+1}
$$


Uma outra forma de interpretar o diagrama de Nyquist é como a parametrização pela frequência $\omega$ de $G(j \omega)$, isso ilustra uma desvantagem dessa representação em relação a outras representações gráficas, pois não se tem informação direta sobre a frequência no gráfico como se tem nos diagramas de Bode, por exemplo. A curva de Nyquist apresenta como característica a simetria em relação ao eixo real, e é muito comum ao se traçar esses diagramas computacionalmente encontrarmos a figura

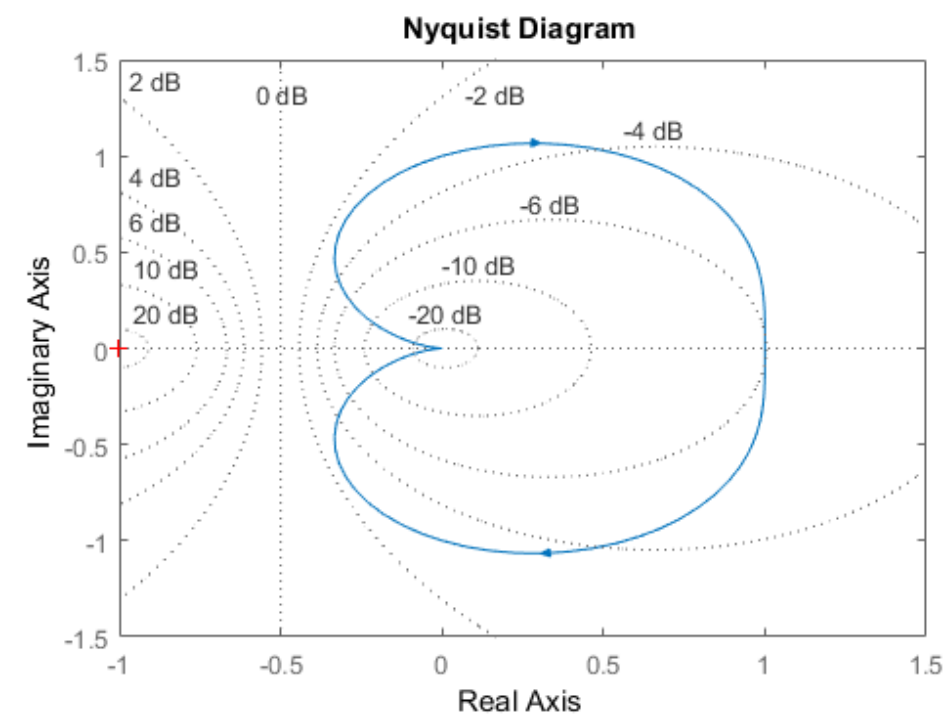

Figura 2.3: Diagrama de Nyquist com a Parte Espelhada

As formas de se obter as curvas são discutidas com riqueza de detalhes na literatura clássica de controle (Ogata 2009), (Franklin et al. 2013) e (Skogestad \& Postlethwaite 2007). Neste trabalho os traçados de Nyquist foram obtidos com o auxílio do MATLAB R2016b.

\subsubsection{Critério de Estabilidade de Nyquist}

O critério de estabilidade de Nyquist determina a estabilidade de um sistema em malha fechada com base na resposta em frequência do sistema em malha aberta. Para um sistema em malha fechada descrito pela seguinte função de transferência:

$$
G_{m f}(s)=\frac{C(s)}{R(s)}=\frac{G(s)}{1+G(s)}
$$

a equação característica é dada por

$$
1+G(s)=0
$$

e para estabilidade, todas as raízes desta equação devem ter parte real negativa, ou seja, estarem situadas no semiplano esquerdo (SPE) do plano s. O critério de estabilidade de Nyquist relaciona o número dessas raízes situadas no semiplano direito (SPD) com o 
número de polos de malha aberta no semiplano direito e o número de envolvimentos do ponto $(-1,0)$ através da equação:

$$
Z=N+P
$$

onde $Z$ representa o número de polos de malha fechada situados no SPD, $N$ o número de envolvimentos do ponto $(-1,0)$, no sentido horário, pelo traçado de Nyquist do ganho de malha de $G(j \omega)$ e $P$ o número de polos de malha aberta no semi-plano direito de $G(s)$. O número $N$ pode ser negativo ou positivo a depender do sentido do envolvimento, para cada enlace no sentido horário a contribuição em $N$ é positiva e para cada enlace no sentido anti-horário a contribuição negativa. Toda essa argumentação é baseada no teorema de Cauchy (teorema do mapeamento complexo) que relaciona a curva no domínio da função com o domínio $s$. Ogata (2009) resume a análise de estabilidade para um sistema por resposta em frequência segundo os critérios de Nyquist em três possíveis casos:

1. Não existir nenhum envolvimento do ponto $-1+j 0$. Implicando em que o sistema será estável se não houver polos de malha aberta no SPD do plano $s$, e instável caso contrário;

2. Existir um ou mais envolvimentos do ponto $-1+j 0$ no sentido anti-horário. Implicando que, para o sistema estável, nesse caso, o número de envolvimentos tem que ser igual ao número de polos de malha aberta no SPD;

3. Existir um ou mais envolvimentos do ponto $-1+j 0$ no sentido horário, implicando nesse caso em instabilidade.

Baseado no teorema do mapeamento, Franklin et al. (2013) resume em quatro passos básicos o processo de determinação de estabilidade de um sistema pelo critério de estabilidade de Nyquist:

1. Obter o diagrama de Nyquist do ganho de malha do sistema, geralmente representado por 2.35;

2. Avaliar o número de envolvimentos do ponto $-1+j 0$ no sentido horário $(\mathrm{N})$, se o envolvimento for no sentido anti-horário o número é negativo;

3. Determinar o número de polos instáveis em malha aberta $(\mathrm{P})$;

4. Calcular o número de raízes instáveis em malha fechada: $Z=N+P$

A vantagem de se utilizar a análise de estabilidade por resposta em frequência utilizando o critério de estabilidade de Nyquist é que o número de polos instáveis de malha fechada (Z) não precisa necessariamente ser determinado, desta forma não há necessidade de se expressar matematicamente o atraso por meio de uma função de transferência de ordem finita, o que dispensa a utilização de aproximações. 


\section{Capítulo 3}

\section{Formulação do Problema}

O sistema matricial de equações diferenciais de $2^{\circ}$ grau mostrado em (3.1), aparece frequentemente em uma gama variada de aplicações em análises estruturais e de vibrações como em Balas (1982) e Vandervelde (1986), onde $\mathbf{M} \in \mathfrak{R}^{n \times n}$, é uma matriz de massas, na maioria dos casos definida positiva; $\mathbf{D} \in \mathfrak{R}^{n \times n}$ é uma matriz de amortecimento, geralmente, pelo menos, semidefinida positiva; $\mathbf{K} \in \mathfrak{R}^{n \times n}$ é uma matriz de rigidez, também sendo semidefinida positiva na maior parte das vezes; $\mathbf{B} \in \mathfrak{R}^{n \times m}$ é uma matriz de controle (também vista como matriz de influência ou de atuadores) de posto completo; $\mathbf{x} \in \mathfrak{R}^{n}$ é o vetor de estado, que para sistemas mecânicos como os da Figura 2.1, representa o deslocamento e $\mathbf{u} \in \mathfrak{R}^{m}$ é um vetor de entradas.

$$
\mathbf{M} \ddot{x}(t)+\mathbf{D} \dot{x}(t)+\mathbf{K} x(t)=\mathbf{B} u(t-\tau)
$$

Assume-se a lei de controle por realimentação de estados como:

$$
u(t-\tau)=\mathbf{f}^{T} \dot{\mathbf{x}}(t-\tau)+\mathbf{g}^{T} \mathbf{x}(t-\tau)
$$

em que f, $\mathbf{g} \in \mathfrak{R}^{n \times m}$ são os vetores de ganho do controlador. Substituindo a equação (3.2) na equação (3.1) e realizando os procedimentos presentados na seção 2.2.2, aplicando a fórmula de Sherman-Morrison, chega-se a matriz de receptância dada por:

$$
\hat{\mathbf{H}}(s)=\mathbf{H}(s)+\frac{\mathbf{H}(s) \mathbf{B}(\mathbf{g}+s \mathbf{f})^{T} \mathbf{H}(s) e^{-s \tau}}{1-(\mathbf{g}+s \mathbf{f})^{T} \mathbf{H}(s) \mathbf{B} e^{-s \tau}}
$$

Desta forma o problema de alocação de autovalores para a matriz de receptância de malha fechada da equação (3.3) pode ser resolvido encontrando-se valores de $\mathbf{g}$ e f solução da equação (2.32). Trabalhos relacionados ao controle em malha fechada aplicados a sistemas de segunda ordem têm ganhado cada vez mais notoriedade devido a sua relevância prática. A consideração do atraso de transporte nestes tipos de sistema é parte indispensável quando se deseja uma representação fidedigna dos modelos levantados. A inserção do atraso em um sistema de segunda ordem origina um QEP que possui infinitas soluções, o que pode acarretar em erro na solução do problema de alocação de autovalor para este sistema. Essa particularidade requer uma análise a posteriori na maioria das abordagens para solução destes problemas. 


\subsection{O Problema da Alocação Indesejada}

Em Ram et al. (2009) o problema de alocação de autovalores para um sistema com atraso é solucionado com a abordagem por matriz de receptância. O método utilizado, discutido na seção 2.2.3 falha na tentativa de alocar um conjunto de autovalores no exemplo numérico para um sistema com grau de liberdade singular mostrado na figura 3.1. Nesse exemplo os valores de $\mathbf{M}=1, \mathbf{D}=0,01, \mathbf{K}=5, \mathbf{B}=1$ e $\tau=0,1$. Supondo que se deseja alocar dois autovalores do sistema para $s_{1}=-1$ e $s_{2}=-47$, seguindo os procedimentos descritos na seção 2.2.3, solucionando a equação (2.32), encontra-se os seguintes valores para os vetores de ganho $\mathbf{f}=-0,3198$ e $\mathbf{g}=5,1001$.

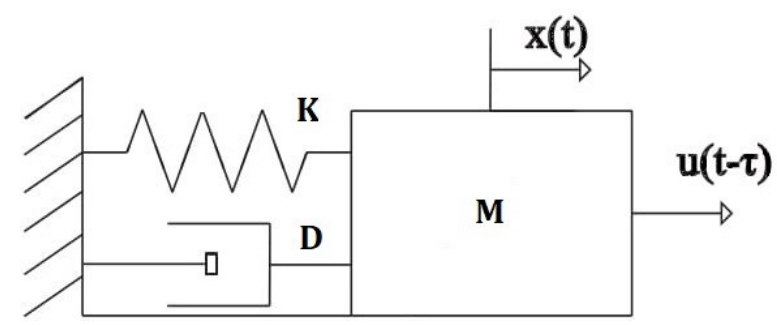

Figura 3.1: Sistema de Segunda Ordem com Atraso - 1 Grau de Liberdade

Substituindo os valores encontrados de f e $\mathbf{g}$ na equação característica do sistema

$$
Q(s)=\left(s^{2} \mathbf{M}+s\left(\mathbf{D}-\mathbf{B f}^{T} e^{-s \tau}\right)+\left(\mathbf{K}-\mathbf{B g}^{T} e^{-s \tau}\right)\right)
$$

plota-se a curva de $Q(s)$ para $-50<s<20$ na Figura 3.2. No gráfico da Figura 3.2 destacam-se os pontos nos quais $Q(s)=0$. Além dos desejados $s=-1$ e $s=-47$ vê-se que $s=0,1065$ também é raiz da equação característica, e, nesta situação muito indesejado pois está situado no semi-plano direito do plano complexo causando instabilidade. Isto demonstra a necessidade indispensável de uma análise a posteriori do vetor de ganho calculado.

\section{Autovalores primários e secundários}

Como discutido na seção 2.2.3 para um sistema sem atraso a definição de $2 n$ autovalores regula completamente a dinâmica do sistema. Considerando a situação limite para o caso sem atraso $(\tau \rightarrow 0)$, o sistema ainda possui um conjunto infinito de autovalores mas somente $2 n$ destes influenciam no comportamento dinâmico do sistema, e a esses autovalores denominamos autovalores primários. Os outros infinitos autovalores, nessa situação limite, não tem influência na dinâmica do sistema e são classificados como autovalores secundários. À medida que a constante de atraso aumenta de zero até um valor constante definido, os $2 n$ autovalores primários mudam de posição no plano $s$ e os outros infinitos autovalores secundários, que na situação limite tinham $\mathfrak{R} \rightarrow-\infty$ pois não influenciavam na dinâmica do sistema, tornam-se finitos. Dessa forma fica difícil diferenciar os autovalores, e também garantir que os autovalores desejados sejam alocados como primários levando a um novo problema na análise a posteriori. 


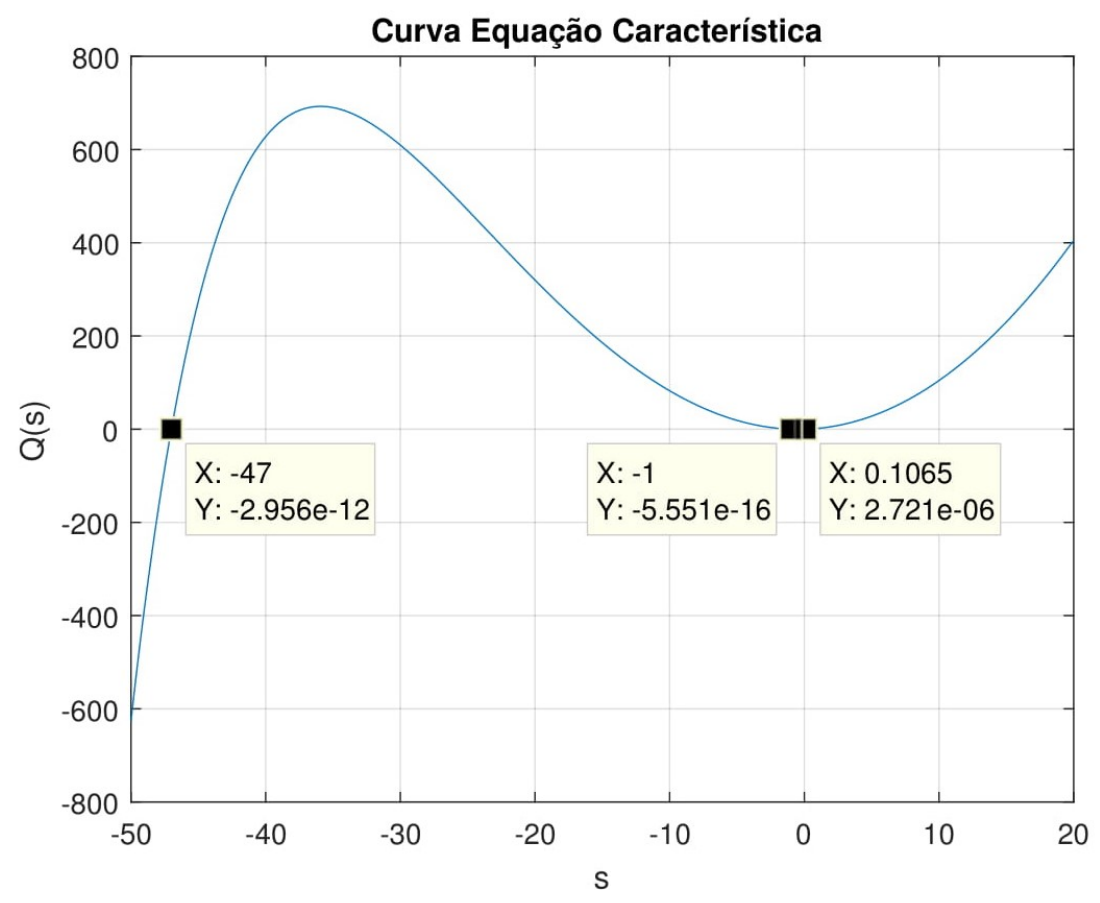

Figura 3.2: Curva da Equação Característica do Sistema da Figura $Q(s)$

A solução proposta por Ram et al. (2009) para o problema de instabilidade causada na alocação para o exemplo da figura 3.1 começa por classificar os grupos de autovalores do sistema e utilizar uma ideia semelhante à alocação parcial de autovalores como visto em Datta et al. (1997). Os autovalores são classificados em primários e secundários em função da influência desses na dinâmica do sistema quando a constante de atraso tende a zero. Para essa classificação a expansão por série de Taylor é utilizada para aproximar os autovalores primários em Ram et al. (2009) obtendo-se melhores resultados à medida que se utilizam expansões de ordens mais elevadas, o que de certa forma torna o trabalho mais difícil, uma vez que, uma expansão de Taylor de segunda ordem leva a um problema de autovalor cúbico.

\subsection{Abordagem Usando o Preditor de Smith}

Em Araújo \& Santos (2018) o controle com atraso é proposto com uma abordagem baseada no Preditor de Smith com o objetivo de compensar o atraso fornecendo um polinômio característico sem o termo exponencial. A predição é obtida utilizando também a abordagem por receptância na obtenção do preditor. A proposta apresenta excelentes resultados para os casos de sistemas com pouco, ou quase nenhum amortecimento, casos como estes em que o sistema tem polos sobre, ou muito próximos do eixo imaginário, trazem dificuldade para se trabalhar com resposta em frequência. 


\subsection{A Ideia da Abordagem por Resposta em Frequência}

Em Araújo (2018a) é feita uma discussão com relação aos resultados apresentados em Ram et al. (2009). A aproximação de Padé é utilizada na aproximação dos autovalores primários, e uma análise qualitativa é feita utilizando a teoria de controle clássico através do levantamento do diagrama de Nyquist do sistema e das margens de ganho e de fase. Com objetivo de comparação, também são obtidos resultados utilizando a metodologia proposta em Ram et al. (2009) para o mesmo exemplo da figura 3.1 com realimentação derivativa. As margens de ganho são relacionadas à alocação e o critério de Nyquist utilizado para análise de estabilidade. Essa ideia deu forma à motivação da proposta apresentada neste trabalho utilizando o ganho de malha do sistema representado pela matriz de receptância.

\subsection{Abordagem do Problema no Domínio da Frequência}

Como ilustrado em Araújo (2018a), o desempenho do sistema e sua estabilidade podem ser aferidos por sua resposta em frequência sem necessidade de aproximações. Dentre as representações em frequência existentes, a escolhida para se trabalhar neste artigo foi o diagrama polar, ou diagrama de Nyquist, obtido como um gráfico do módulo versus ângulo de fase do sistema em malha aberta, em coordenadas polares, com a frequência $\omega$ variando de zero a infinito. Nesta representação a estabilidade em malha fechada de um sistema pode ser determinada de acordo com o critério de estabilidade de Nyquist, que estabelece que para que um sistema seja estável em malha fechada, o número de voltas da curva de Nyquist do sistema em torno do ponto $(-1,0)$ no plano $j \omega$ no sentido antihorário, deverá ser igual ao número de polos de malha aberta do sistema no semi-plano direito do plano $s$. Assim, para sistemas estáveis em malha aberta, (sem nenhum polo de malha aberta situado no semi-plano direito), não deve haver nenhum envolvimento do ponto $(-1,0)$ pelo traçado de Nyquist, para que em malha fechada o sistema seja estável.

A equação característica da matriz de receptância dada pela equação (3.3) é definida como:

$$
Q(s)=1-(\mathbf{g}+s \mathbf{f})^{T} \mathbf{H}(s) \mathbf{B} e^{-s \tau}=0
$$

e as raízes dessa equação definem os polos de malha fechada do sistema, muito semelhante ao caso clássico de controle por realimentação unitária para sistemas SISO, como mencionado em (Araújo 2018a), com destaque para o ganho de malha, que neste caso é definido como:

$$
L(s)=-(\mathbf{g}+s \mathbf{f})^{T} \mathbf{H}(s) \mathbf{B} e^{-s \tau}
$$

A partir do ganho de malha do sistema, pode-se levantar o diagrama de Nyquist de $L(j \omega) \mathrm{e}$ analisar o comportamento do sistema por sua resposta em frequência. Para cada conjunto de ganhos de controlador, $\mathbf{g} e \mathbf{f}$, tem-se uma resposta diferente, e pode-se formular $\mathrm{o}$ problema de controle como encontrar valores para os vetores de ganho que atendam a pré-requisitos de projeto no domínio da frequência. 


\subsection{Parâmetro de Projeto e Formulação Matemática}

\section{Circunferência $\mathbf{M}_{s}$}

Margens de ganho e de fase são usualmente utilizadas como parâmetros de projeto de controladores por resposta em frequência. As relações entre a resposta temporal e essas margens fornecem boas referências, ainda que dadas de maneira indireta, para o controle de sistemas via resposta em frequência. Em algumas referências essas margens são chamadas de margens de estabilidade, muito embora para casos com sistemas com mais de um grau de liberdade essas não sejam medidas de estabilidade muito precisas.

Dadas as discussões anteriores sobre estabilidade com a definição do critério de estabilidade de Nyquist, uma conclusão para sistemas estáveis em malha aberta, ou seja, sem nenhum polo de malha aberta no SPD $(P=0)$, é que não se deve haver nenhum envolvimento do ponto $(-1,0)$ pelo diagrama de Nyquist do sistema. Assim, surge a ideia de se definir como parâmetro de projeto uma medida que garanta estabilidade visando atender a este critério. Skogestad \& Postlethwaite (2007) em uma discussão acerca de critérios de picos máximos trazem a ideia da relação entre o chamado pico máximo da função de sensibilidade e a menor distância entre o ponto $(-1,0)$ e o diagrama de Nyquist do ganho de malha do sistema. Baseado nesse conceito, define-se uma circunferência de raio $\mathbf{M}_{s}^{-1}$ centrada no ponto $-1+j 0$ delimitando a região na qual o traçado de Nyquist é permitido, assim, garantindo uma distância segura do ponto $(-1,0)$, ilustrando a ideia de sistema robusto.

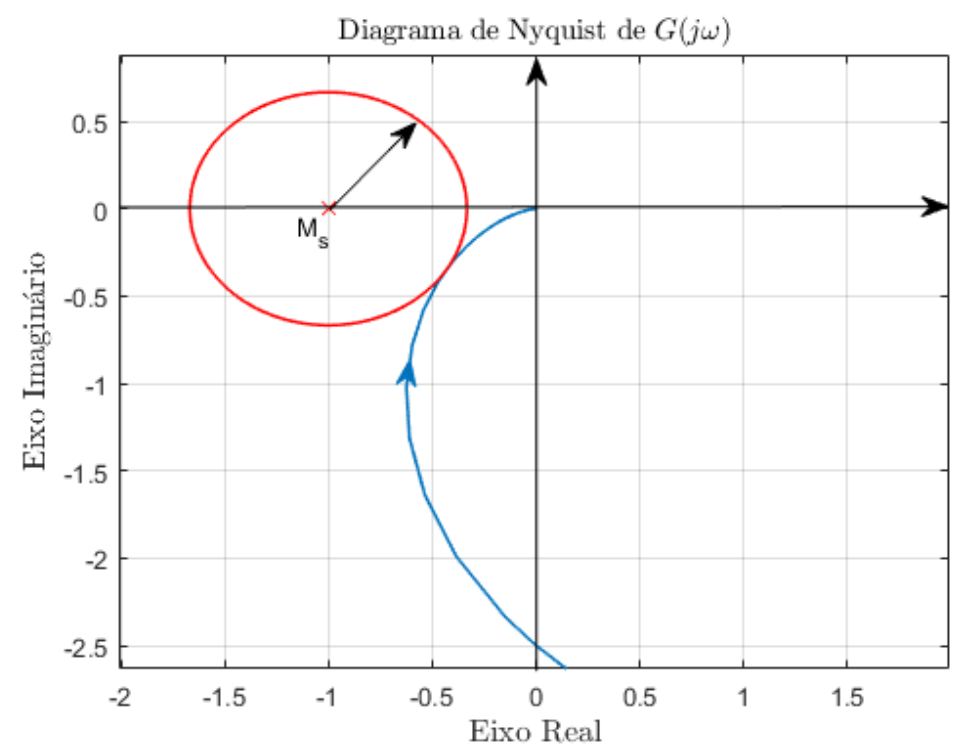

Figura 3.3: Diagrama de Nyquist Exemplo de Circunferência $\mathrm{M}_{s}$

\section{Definição do problema de otimização}

A figura 3.3 mostra o diagrama de Nyquist de um sistema genérico (traçado em azul) e uma circunferência (em vermelho) representando a circunferência $\mathrm{M}_{s}$. À medida que o 
ganho do sistema aumenta o diagrama de Nyquist invande a área da circunferência e se aproxima do ponto $-1+j 0$ chegando próximo da fronteira da instabilidade. Com essa ideia, define-se como parâmetro de projeto que o sistema tenha uma distância mínima do ponto $(-1,0)$ definida pelo raio dessa circunferência. A solução apresentada neste trabalho visa garantir uma estabilidade robusta, a fim de garantir que mesmo sujeito a alterações de parâmetros o sistema seja estável. Para isso deve-se manter o ponto $(-1,0)$ a uma distância segura de $L(j \omega)$, o que pode ser interpretado como o traçado de $L(j \omega)$ fora de uma circunferência centrada em $(-1,0)$ cujo raio seria a distância mínima desejada com o cuidado de não apresentar soluções nas quais as curvas de $L(j \omega)$ estejam muito afastadas do circulo, o que levaria a respostas muito lenta. Dessa forma, busca-se fazer com que a curva de $L(j \omega)$ tangencie o circulo $\mathrm{M}_{s}$ estabelecido e então o seguinte problema de otimização pode ser formulado:

$$
\begin{array}{ll}
\min _{\mathbf{f}, \mathbf{g}} & h(\mathbf{f}, \mathbf{g})=\left(\min _{\omega_{i}}|L(j \omega)+1|-\mathbf{M}_{s}^{-1}\right)^{2} \\
\text { s.a. } & L\left(j \omega_{i}\right)=-\left(\mathbf{g}+j \omega_{i} \mathbf{f}\right)^{T} \mathbf{H}\left(j \omega_{i}\right) \mathbf{B} e^{-j \omega_{i} \tau} \\
& \operatorname{Re}\left\{L\left(j \omega_{i}\right)\right\} \geq-1+\mathbf{M}_{s}^{-1} \forall \omega_{i} / \operatorname{Im}\left\{L\left(j \omega_{i}\right)\right\}=0
\end{array}
$$

onde o subscrito $i$ indica o $i$-ésimo valor de frequência com $\omega$ variando de zero a um valor elevado o suficiente para que $\left|L\left(j \omega_{i}\right)\right| \simeq 0$. A equação (3.6) estabelece o problema de minimização da função genérica $h(\mathbf{f}, \mathbf{g})$, que por ser uma função quadrática terá seu valor mínimo para $\left(\min _{\omega_{i}}|L(j \omega)+1|=\mathrm{M}_{s}^{-1}\right)$, e como $\left(\min _{\omega_{i}}|L(j \omega)+1|\right)$ é a menor distância entre $L(j \omega)$ e o ponto $(-1,0)$, a igualdade significa que o valor mínimo para o problema dado pela equação (3.6) se dará num ponto tangente a circunferência de raio $\mathrm{M}_{s}^{-1}$.

\subsection{Otimização de Desempenho}

O problema de otimização definido matematicamente pela equação (3.6) estabelece a busca por um conjunto de vetores de ganho de controlador que garantam que a curva de Nyquist do sistema de segunda ordem não adentre a região delimitada pela circunferência centrada no ponto $(-1,0)$, de raio $\mathbf{M}_{s}^{-1}$. A restrição dada pela equação (3.7) garante que soluções como a de um conjunto solução aleatório mostrado na figura 3.4 não sejam obtidas pela busca, pois como ilustrado, a curva de Nyquist da figura 3.4 enlaça o ponto $(-1,0)$, ilustrando instabilidade.

O problema de otimização dado pela equação (3.6) não garante todavia que o desempenho da resposta seja levada em consideração de forma direta, apesar da ideia de robustez, não se garante respostas rápidas tão quanto desejadas somente com a definição da região "proibida"para o traçado da curva de Nyquist de $L(j \omega)$. Em alguns trabalhos na literatura o desempenho do sistema é abordado utilizando-se técnicas de ajuste de curva, sintonia de ganhos do controlador, integral do erro e itens relacionados a resposta temporal (Euzébio \& Barros 2015, Euzébio \& Barros 2013). 


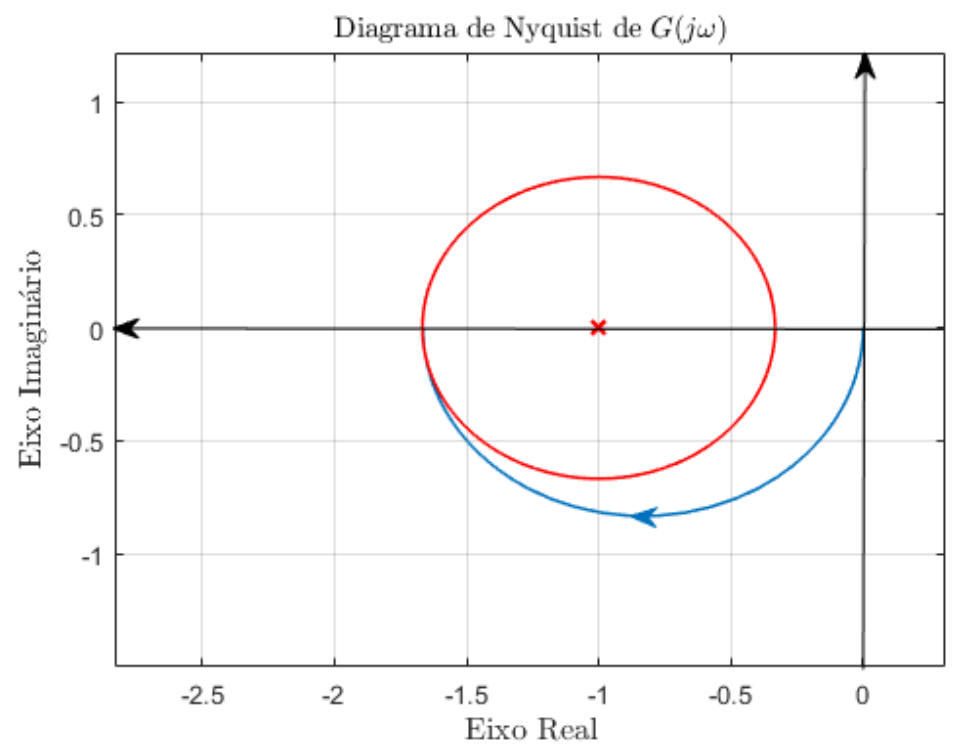

Figura 3.4: Diagrama de Nyquist de um Sistema Genérico para Exemplo de um Caso de Instabilidade

\section{Restrição adicional}

Em Skogestad \& Postlethwaite (2007) é visto que as margens de ganho e de fase do sistema têm uma relação direta com a circunferência $\mathrm{M}_{s}$. A definição dessa circunferência como parâmetro de projeto por resposta em frequência garante um mínimo de margem de ganho e margem de fase, o que torna redundante essas especificações uma vez definido o valor de $\mathrm{M}_{s}$ desejado. Skogestad ainda define matematicamente que:

$$
G M \geq \frac{\mathrm{M}_{s}}{\mathrm{M}_{s}-1} ; \quad P M \geq 2 \operatorname{arcsen}\left(\frac{1}{2 \mathrm{M}_{s}}\right) \geq \frac{1}{\mathrm{M}_{s}}[\mathrm{rad}]
$$

sendo GM a margem de ganho ("gain margin") e PM a margem de fase ("phase margin"). Da equação (3.8) pode-se concluir que, definido um valor $\mathrm{M}_{S}$ garante-se um mínimo valor de $G M$ e $P M$.

Na literatura clássica de controle como em Ogata (2009) e Franklin et al. (2013) a margem de ganho é definida como um fator pelo qual o ganho do sistema pode ser aumentado antes de atingir a instabilidade, e a influência na resposta temporal do sistema é refletida em sua velocidade, respostas mais rápidas são alcançadas para sistemas com menores margens de ganho. Uma vez que se tem definido com $\mathrm{M}_{s}$ um indicativo de robustez, mesmo um mínimo de margem de ganho ainda seria confortável. Assim, a menor margem de ganho (e consequentemente melhor desempenho em termos de velocidade de resposta) se daria para o caso limite em que

$$
G M=\frac{\mathrm{M}_{s}}{\mathrm{M}_{s}-1}
$$

o que significa graficamente que o diagrama de Nyquist do sistema tangencia a circun- 
ferência de raio $\mathrm{M}_{s}$ no ponto sobre o eixo real (uma vez que a margem de ganho é dada como o ganho do sistema quando a fase é igual a $-180^{\circ}$ ), concluindo-se que dentre as soluções para o problema de otimização apresentado pela equação (3.6) as que apresentariam melhores desempenho seriam aquelas que tangenciam a circunferência $\mathbf{M}_{s}$ no ponto da circunferência sobre o eixo real. Nos exemplos apresentados no capítulo 5 essa discussão é ilustrada, e para garantir esse ponto de tangência uma restrição adicional é atribuída ao problema (3.6), sendo formulado novamente da seguinte maneira:

$$
\begin{array}{ll}
\min _{\mathbf{f}, \mathbf{g}} & h(\mathbf{f}, \mathbf{g})=\left(\min _{\omega_{i}}|L(j \omega)+1|-\mathbf{M}_{s}^{-1}\right)^{2} \\
\text { s.a. } & L\left(j \omega_{i}\right)=-\left(\mathbf{g}+j \omega_{i} \mathbf{f}\right)^{T} \mathbf{H}\left(j \omega_{i}\right) \mathbf{B} e^{-j \omega_{i} \tau} \\
& \operatorname{Re}\left\{L\left(j \omega_{i}\right)\right\} \geq-1+\mathbf{M}_{s}^{-1} \forall \omega_{i} / \operatorname{Im}\left\{L\left(j \omega_{i}\right)\right\}=0 \\
\min _{\omega_{i}} & \operatorname{Re}\left\{L\left(j \omega_{i}\right)\right\}=-1+\mathbf{M}_{s}^{-1} \forall \omega_{i} / \operatorname{Im}\left\{L\left(j \omega_{i}\right)\right\}=0
\end{array}
$$

A restrição da equação (3.9) garante que o menor valor dentre os valores reais da curva de Nyquist de $L(j \omega)$ cuja parte imaginária é igual a zero seja igual ao raio da circunferência $\mathrm{M}_{s}-1$, que é a distância entre a origem e o ponto da circunferência sobre o eixo imaginário, garantindo assim, que o diagrama de Nyquist tangencie a circunferência neste ponto. 


\section{Capítulo 4}

\section{Implementação do Método Proposto}

Este capítulo visa apresentar o método desenvolvido para se alcançarem os resultados apresentados no Capítulo 5. A busca pela solução para o problema de otimização descrito no Capítulo 3 é feita usando algoritmo genético, um algoritmo estocástico de base populacional que pesquisa aleatoriamente por mutação e cruzamento entre os membros da população, que pode ser utilizado na solução de problemas de otimização suaves ou não-suaves com restrições lineares ou não lineares (MathWorks 2019, Goldberg \& Holland 1988).

\subsection{Solução do Problema de Otimização}

Uma das alternativas na solução de problemas de otimização, como o descrito na equação (3.6), é o algoritmo genético (GA), um método para solução de problemas de otimização com ou sem restrições baseado no processo de seleção natural que imita a evolução biológica, pioneiro na solução de problemas do tipo abordado neste trabalho (MathWorks 2019). O algoritmo de forma repetitiva modifica a população de soluções individuais. Em cada passo, o algoritmo genético seleciona indivíduos aleatoriamente da população atual e os usa como pais para produzir filhos para a próxima geração. Depois de sucessivas gerações, a população evolui para uma solução ótima. O que atende perfeitamente ao objetivo de busca para o problema descrito pela equação (3.9). Pode-se utilizar o algoritmo genético para solucionar problemas que não são bem adequados para algoritmos de otimização padrão, incluindo problemas em que a função objetivo é descontínua, não-diferenciável, estocástica, ou altamente não linear, como é o caso tratado aqui, sendo a função objetivo abordada pela curva de Nyquist do sistema para valores desejados de $\mathbf{f}$ e g.

\section{Algoritmo genético do MATLAB}

Na solução do problema apresentado neste trabalho optou-se por trabalhar com o algoritmo genético disponível na "toolbox" de otimização do MATLAB versão R2016b. Utilizando a função $g a$ foi escrito um algoritmo, seguindo os passos do Algoritmo 1, que realiza a busca por vetores de ganho $\left[\begin{array}{ll}\mathbf{g} & \mathbf{f}\end{array}\right]$ solução para o problema de otimização da equação (3.6). Essa função requer como parâmetros de entrada obrigatórios uma função 
objetivo e o número de variáveis da resposta, ou o tamanho do vetor solução. Além destas, são acrescentadas uma função de restrição para descrever as restrições dadas pelas equações (3.7) e (3.9) e uma classe de opções de sintonia e inicialização do algoritmo "options".

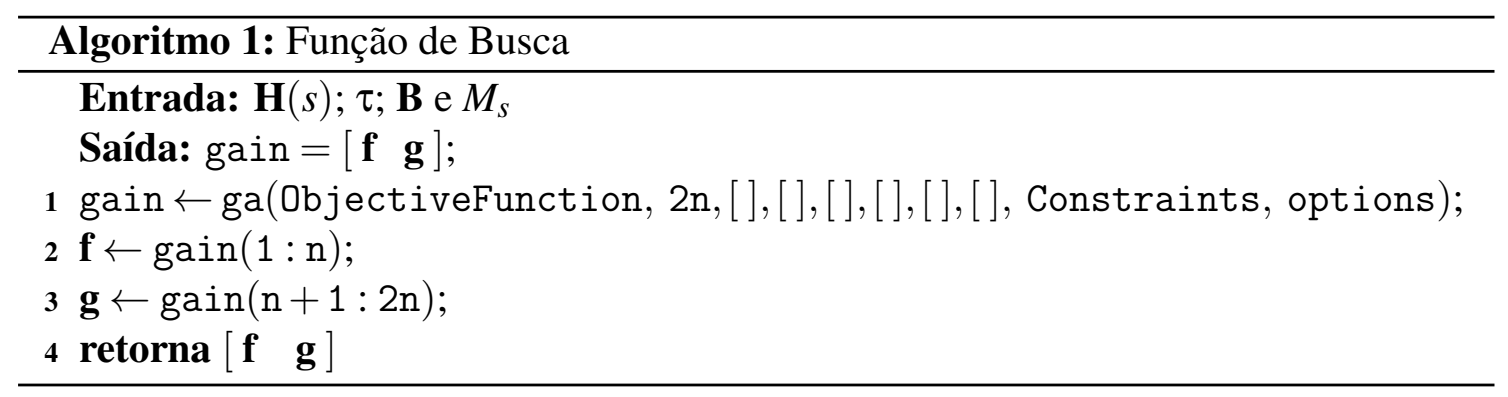

\section{População inicial}

O passo inicial para inicializar um algoritmo genético é a população inicial. Essa denominação, assim como todas as demais relacionadas com o algoritmo, é baseada na analogia com a teoria da evolução biológica, e no problema de otimização define o conjunto inicial de possíveis soluções para o problema definido. Neste trabalho optou-se pela utilização do algoritmo disponibilizado pelo MATLAB versão R2016b que gera aleatoriamente um conjunto de soluções possíveis para o problema ou aceita a definição de um vetor como parâmetro de entrada. Na solução do problema apresentado pela equação (3.6) definir uma população inicial direciona a busca para que haja convergência para a reposta desejada poupando tempo de execução do algoritmo e garantindo soluções satisfatórias.

\section{Procedimento baseado em "detuning"}

A restrição do problema descrita pela equação (3.7) visa garantir a estabilidade do sistema para a resposta encontrada pela busca do algoritmo genético. Em alguns casos, como para sistemas sem amortecimento, em que os polos de malha aberta encontram-se sobre o eixo imaginário, é difícil de se trabalhar com resposta em frequência dadas as descontinuidades geradas por esses pontos na relação entre os planos $(s$ e $j \omega)$. Isso pode refletir na resposta retornada pela busca do " $g a$ " como um mínimo local ou como solução inexistente para o problema descrito sujeito às restrições apresentadas. Motivado por essa dificuldade e baseado na ideia apresentada para sistemas com atraso variável, apresentada em Santos et al. (2018), cria-se uma população inicial onde todos os indivíduos dessa população apresentam soluções estáveis.

O procedimento para geração da população inicial é realizado da seguinte maneira. Primeiro define-se um conjunto de vetor de ganho $[\mathbf{f} \mathbf{g}]$, aqui não há restrições quanto a definição desse vetor, uma sugestão pode ser o vetor calculado pelo método definido em Mottershead \& Ram (2007) para sistemas sem atraso ou simplesmente um ganho unitário. Depois verifica-se a estabilidade do sistema para o ganho de malha 3.5 com os valores pre-estabelecidos para $\left[\begin{array}{ll}\mathbf{f} & \mathbf{g}\end{array}\right]$ no passo anterior. Caso o sistema seja instável multiplica-se 
o vetor $[\mathbf{f} \mathbf{g}]$ por um valor $0 \leq \alpha \leq 1$ e novamente verifica-se a estabilidade para $L(s)$ com $\alpha[\mathbf{f} \mathbf{g}]$ e vai reduzindo o valor de $\alpha$ até que se atinja a estabilidade e a partir daí constrói-se o vetor de população inicial como no algoritmo2.

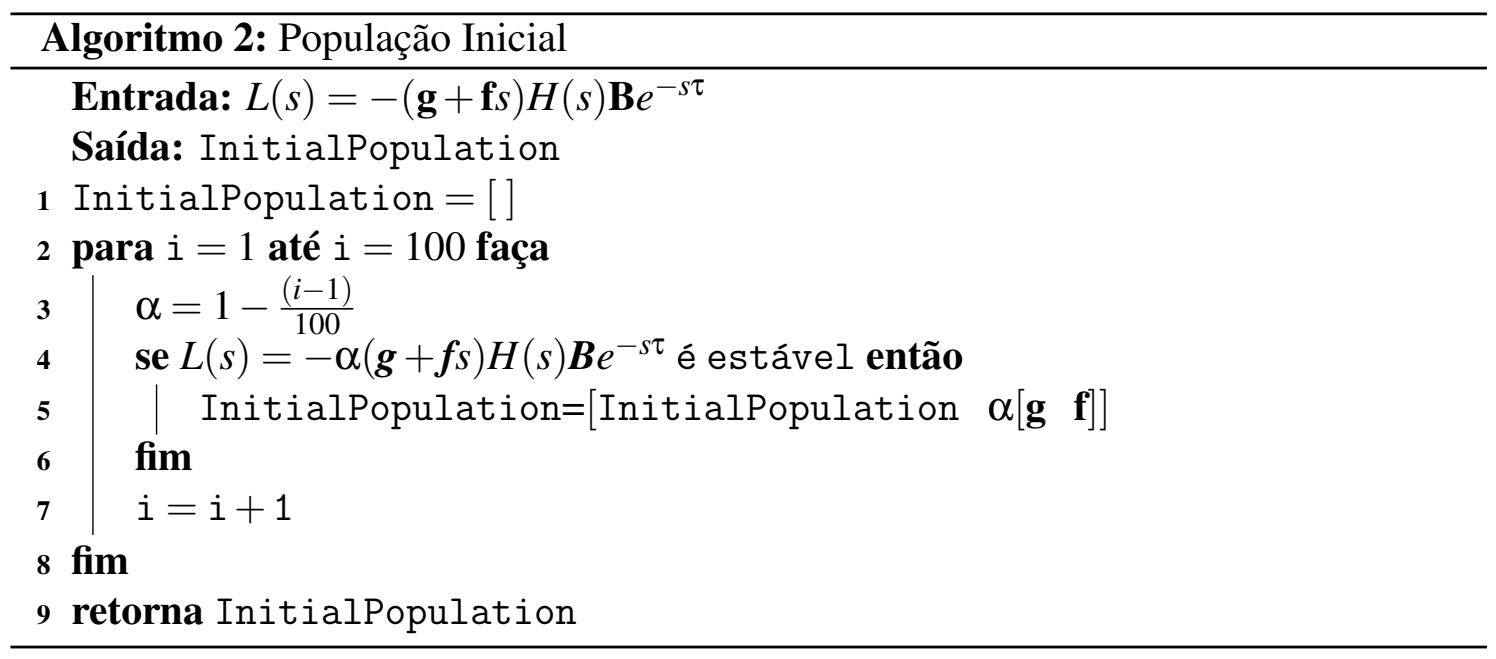

\subsection{A Função Objetivo}

A função objetivo descrita pelo Algoritmo 3 descreve o problema de otimização deste trabalho, dado pela equação (3.6). É utilizada como argumento de entrada na função $g a$ (algoritmo genético do MATLAB) e é onde se realiza a avaliação dos indivíduos da população. Isto visa garantir que a menor distância entre o ponto $-1+j 0$ e o diagrama de Nyquist de $L(j \omega)$ seja igual ao raio da circunferência $M_{s}$, definida como parâmetro de projeto na resposta em frequência. A função "nyquist" obtém os valores de $L\left(j \omega_{i}\right)$ e armazena nos vetores Re e Im as respectivas partes real e imaginária de $L\left(j \omega_{i}\right)$. O subscrito $i$ indica o $i$-ésimo valor de frequência $\omega$ e pode ser deixado para a função determinar o intervalo de frequências, ou pode ser definido da construção do algoritmo. O vetor " $d$ " armazena as distâncias entre o ponto $(-1,0)$ e os pontos sobre a curva $L(j \omega)$ armazenadas nos vetores Re e Im. Por fim a função "ff"representa a avaliação do indivíduo da população (candidato a solução) do problema de otimização e esse valor é utiliado na avaliação pelo algoritmo genético ga. 


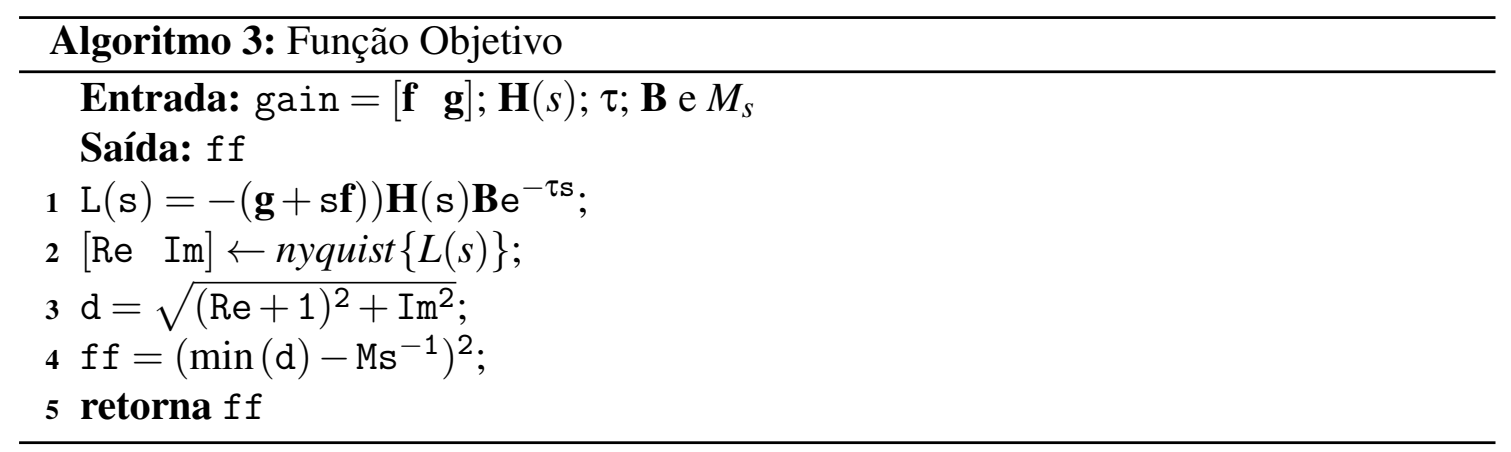

\subsection{A Função de Restrições}

O argumento "Função de Restrição"presente no algoritmo de busca descrito pelo Algoritmo 1 descreve as restrições do problema de otimização dadas pelas equações (3.7) e (3.9). O vetor "cross"armazena todos os cruzamentos da curva de Nyquist de $L(j \omega)$ com o eixo real. A variável "c"representa a restrição não linear dada pela inequação (3.7), sendo $c \leq 0$, e a variável "ceq"representa a restrição não linear dada pela equação (3.9), sendo $c e q=0$.

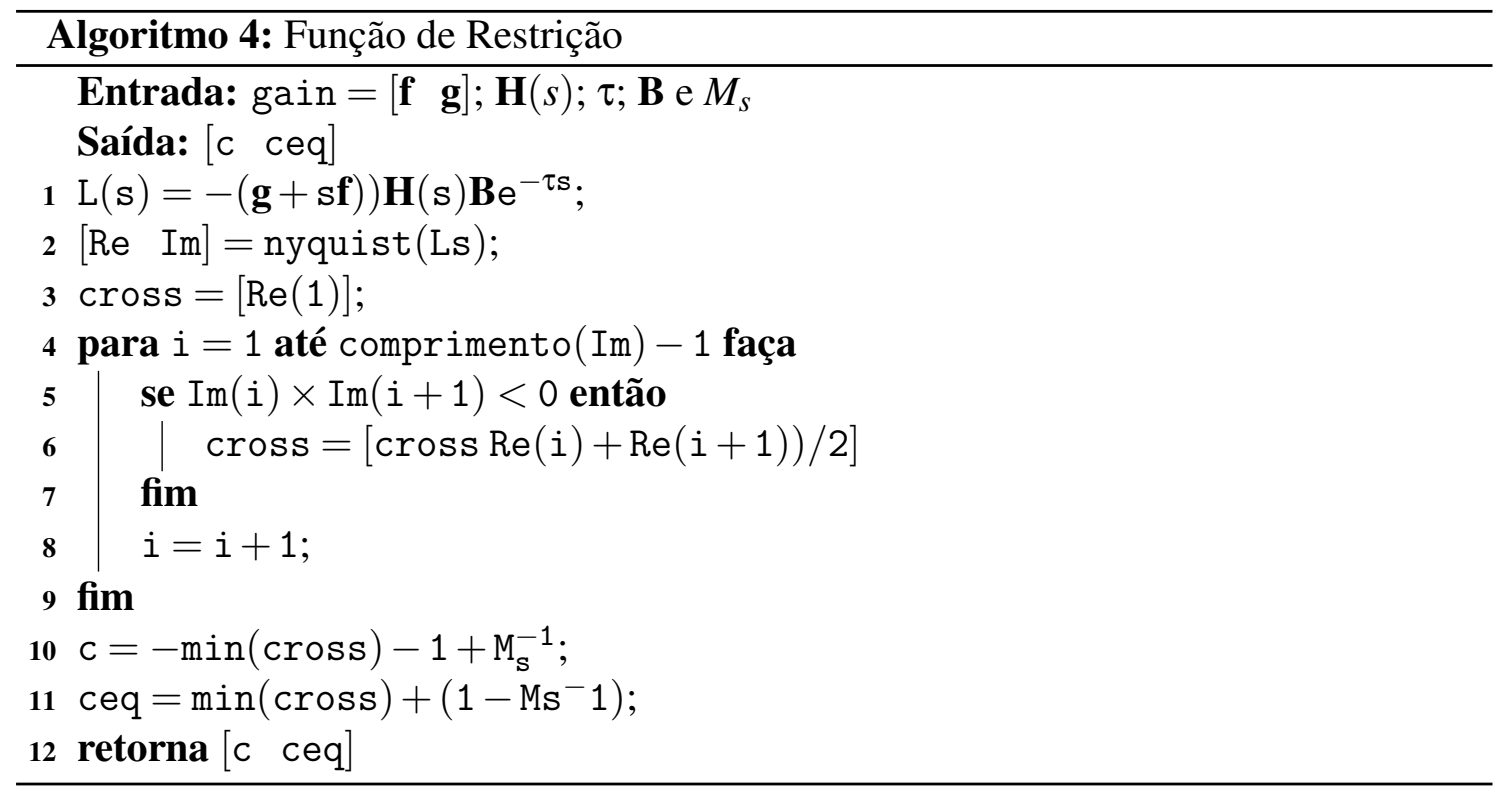

A função de restrição retorna para o $g a$ os valores de "c"e "ceq"e realiza a avaliação do individuo da população juntamente com a função objetivo. $\mathrm{O}$ algoritmo finaliza a busca assim que as mudanças entre as gerações não forem mais significativas, exibindo como resposta o melhor individuo avaliado pelas funções descritas. O capítulo 5 apresenta os resultados obtidos com o algoritmo desenvolvido. 


\section{Capítulo 5}

\section{Experimentos e Resultados}

\subsection{Exemplo 1}

Um exemplo clássico de aplicação é o sistema massa mola amortecedor de um grau de liberdade mostrado na Figura 5.1 .

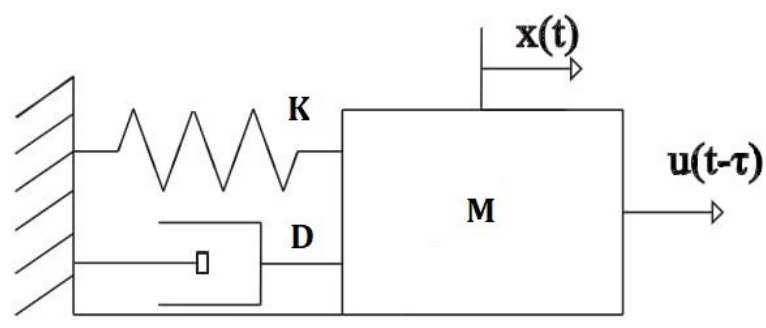

Figura 5.1: Sistema massa-mola-amortecedor de um grau de liberdade

Para $\mathbf{M}=1, \mathbf{D}=0,01, \mathbf{K}=5, \mathbf{B}=1$ e $\tau=0,1$, como no Exemplo 2 de Ram et al. (2009), define-se o problema de controle por realimentação de estados como encontrar valores de $\mathbf{f}$ e g, que garanta que o diagrama de Nyquist de $L(j \omega)$ esteja completamente fora da área compreendida pela circunferência de raio $M_{s}^{-1}$, definida como parâmetro de projeto. Como mencionado anteriormente, a definição de $M_{s}$ estabelece, através de uma relação muito próxima, margens de ganho e de fase para o sistema, tornando redundante as definições dessas margens como parâmetro de projeto. Os valores mais comuns de $M_{S}$ estão dentro do intervalo de 1,22 $\leq M_{S} \leq 1,667$ (Skogestad \& Postlethwaite 2007).

O método apresentado em Ram et al. (2009) visa alocar os polos de malha fechada em $s_{1}=-1$ e $s_{2}=-47$, neste cenário a alocação falha em obter uma resposta estável, o que torna indispensável uma análise à posteriori das soluções de problemas de alocação para sistemas com atraso, para este método. Como alternativa, uma aproximação por série de Taylor para o atraso é proposta por Ram et al. (2009), alcançando melhores resultados para séries de grau mais elevado, em Araújo \& Santos (2018) a proposta de solução passa pela aplicação de um filtro preditor de Smith com o objetivo de eliminar os efeitos do atraso no sistema.

O método aqui proposto aborda os parâmetros de projeto baseados no diagrama de Nyquist do ganho de malha do sistema $(L(j \omega)$, abordando os conceitos de estabilidade 
segundo os critérios de Nyquist. A solução buscada visa atender a um sistema relativamente robusto, com $\mathrm{M}_{s}=1,66$ como objetivo. A Tabela 5.1 resume os valores de ganhos encontrados com a utilização dos métodos citados.

\begin{tabular}{|c|c|c|}
\hline Método & f & g \\
\hline \hline Alocação com Atraso & $-0,3198$ & 5,1001 \\
\hline \hline Alocação sem Atraso & $-47,9900$ & $-42,0000$ \\
\hline \hline Método Proposto SRA & $-2,6097$ & $-5,9599$ \\
\hline \hline Método Proposto CRA & $-4,4300$ & $-6,1675$ \\
\hline \hline
\end{tabular}

Tabela 5.1: Tabela de Ganhos de Realimentação de Estado

Com relação as discussões sobre otimização da resposta temporal feita na seção 3.6 , é feito um comparativo para os vetores obtidos com o método proposto onde há a restrição adicional, sem a restrição adicional, para o método de alocação proposta por Ram et al. (2009) e para o caso em que o atraso é desconsiderado. Para os ganhos obtidos pela Alocação com Atraso, proposta por Ram et al. (2009), e no caso em que o atraso é desconsiderado (Alocação sem Atraso), o sistema fica instável.

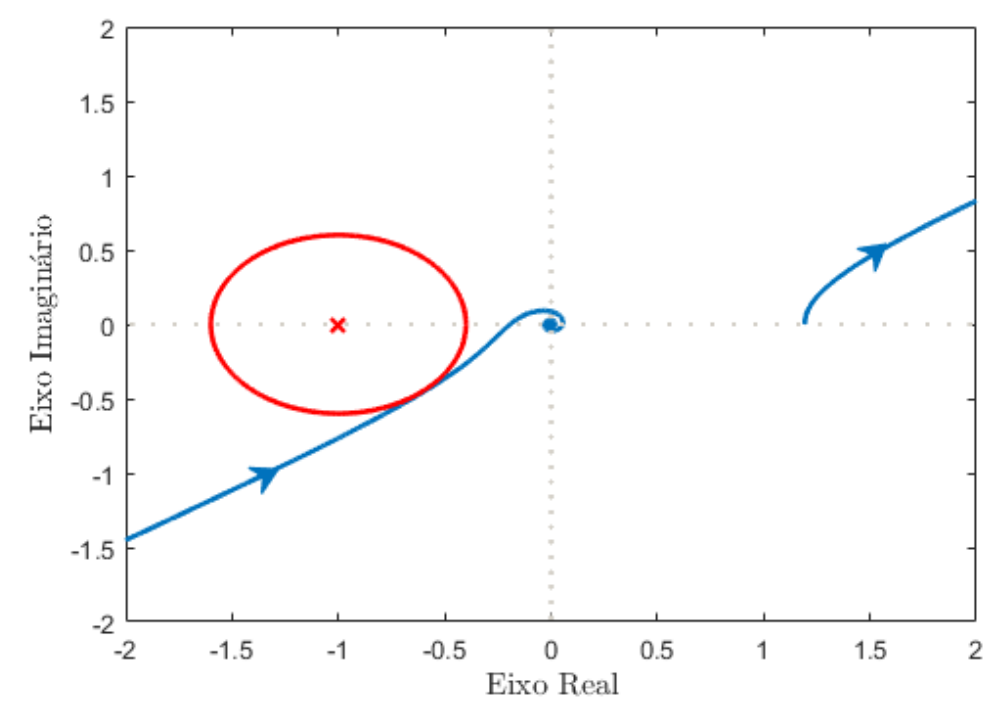

Figura 5.2: Diagrama de Nyquist Ganhos sem Restrição Adicional

A Figura 5.2 mostra o diagrama de Nyquist obtido pelo método proposto para o caso em que não se considera a restrição adicional. O que se observa no traçado da curva é que ela permanece completamente fora da circunferência em vermelho tangenciando o circulo na parte direita inferior, alcançando o objetivo da função do problema de otimização.

A Figura 5.3 mostra o diagrama de Nyquist obtido para o vetor de ganho encontrado pelo método proposto com a restrição adicional de se obter a tangência entre a curva de Nyquist e a circunferência $\mathrm{M}_{s}$ no ponto sobre o eixo real. Em ambos os casos das figuras mencionadas o objetivo de manter a curva de Nyquist de $L(j \omega)$ completamente 


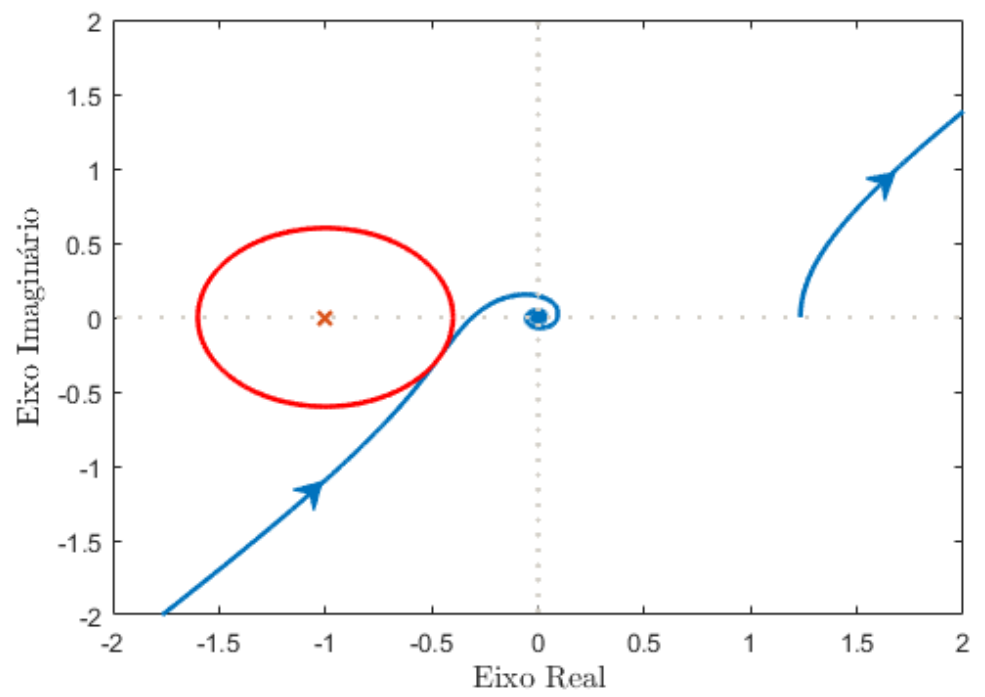

Figura 5.3: Diagrama de Nyquist Ganhos com Restrição Adicional

fora da circunferência $\mathrm{M}_{s}$ foi atendido, as diferenças entre o desempenho temporal das duas respostas são exibidas nas Figuras 5.4 e 5.5, onde são plotadas as respostas do estado $\mathbf{x}(t)$ (posição), para os casos do método proposto com restrição adicional e sem restrição adicional, e para derivada do estado $\dot{\mathbf{x}}(t)$ (velocidade), para condições iniciais $\dot{\mathbf{x}}(0)=0$ e $\mathbf{x}(0)=0,3$.

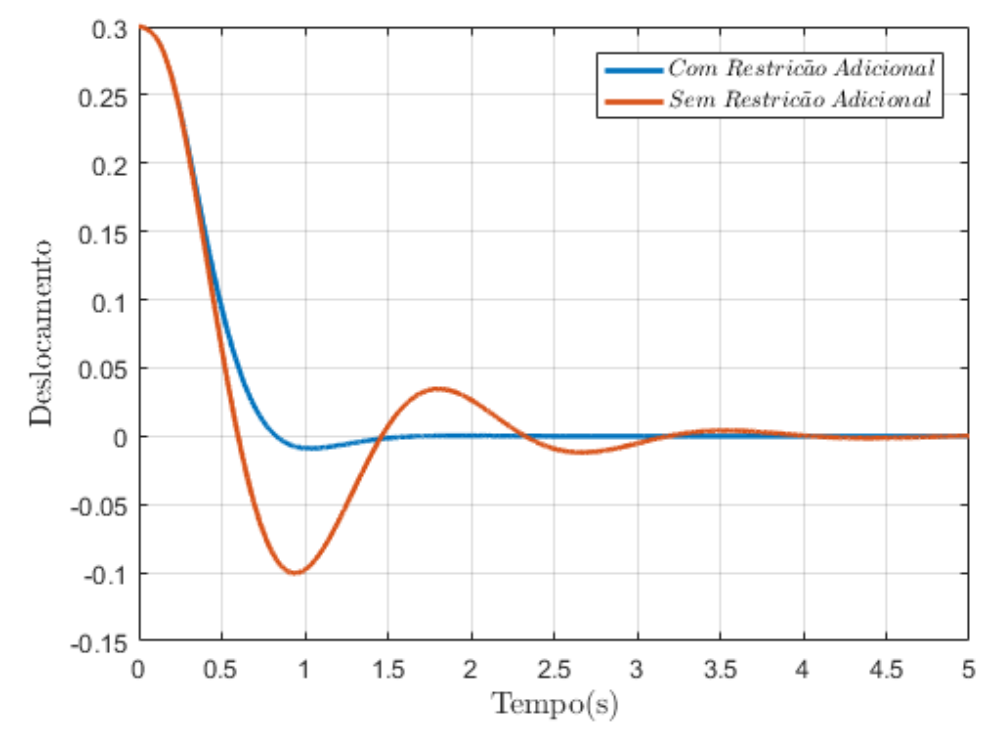

Figura 5.4: Resposta do Estado $\mathbf{x}(t)$ para condições iniciais $\dot{\mathbf{x}}(0)=0$ e $\mathbf{x}(0)=0,3$

Neste exemplo clássico de um grau de liberdade é fácil perceber as características típicas de um sistema de segunda ordem amortecido. Como visto em Skogestad \& Postlethwaite (2007), para um valor de $M_{s}=1,667$, definido como parâmetro para este exem- 


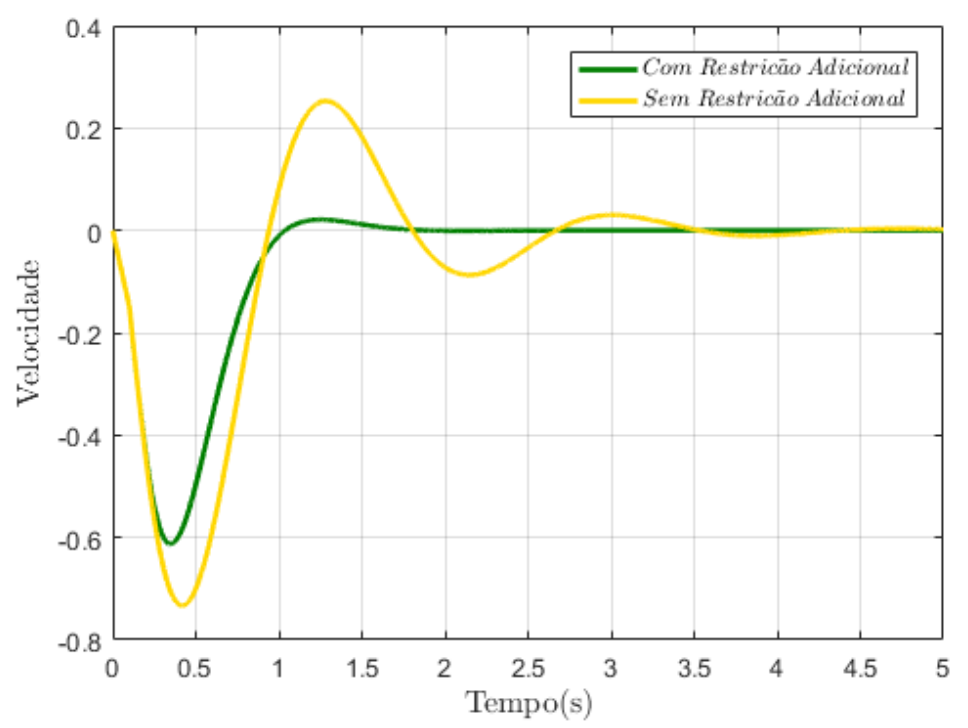

Figura 5.5: Resposta do Estado $\dot{\mathbf{x}}(t)$ para condições iniciais $\dot{\mathbf{x}}(0)=0$ e $\mathbf{x}(0)=0,3$

plo, a relação de coeficiente de amortecimento, por exemplo, se aproxima de $\zeta=0,6$.

$\mathrm{Na}$ Tabela 5.2 são mostrados os valores de margem de ganho (GM) para o sistema do Exemplo 1, com os ganhos de realimentação de estado exibidos na Tabela 5.1. Esses valores podem servir de ilustração para estabilidade do sistema, uma vez que da definição, a margem de ganho indica de quanto o ganho de um sistema pode ser aumentado até que este atinja a instabilidade (Ogata 2009).

\begin{tabular}{|c|c|}
\hline Método & GM \\
\hline \hline Alocação com Atraso & $-0,172 \mathrm{~dB}$ \\
\hline \hline Alocação sem Atraso & $4,26 \mathrm{~dB}$ \\
\hline \hline Método Proposto SRA & $14,3 \mathrm{~dB}$ \\
\hline \hline Método Proposto CRA & $10,2 \mathrm{~dB}$ \\
\hline \hline
\end{tabular}

Tabela 5.2: Margens de ganho

\subsection{Exemplo 2}

Usando como exemplo prático de aplicação do método apresentado, visto em Ram et al. (2011), considere as matrizes de massa, amortecimento e elasticidade como sendo:

$$
\mathbf{M}=\left[\begin{array}{ll}
1 & 0 \\
0 & 1
\end{array}\right], \mathbf{D}=\left[\begin{array}{cc}
1 & -1 \\
-1 & 1
\end{array}\right], \mathbf{K}=\left[\begin{array}{cc}
3 & -2 \\
-2 & 3
\end{array}\right]
$$

Para uma matriz de entradas $\mathbf{B}^{\prime}=\left[\begin{array}{ll}0 & 1\end{array}\right]$ e uma constante de atraso $\tau=5$, os autovalores de malha aberta do sistema são, $\lambda_{1,2}= \pm j$ e $\lambda_{3,4}=-1 \pm 2 j$. O método apresentado em 
Ram et al. (2011) tem como objetivo realocar o primeiro auto-par para $\mu_{1,2}=-1 \pm j$ preservando o segundo. Este exemplo particular mostra um sistema não amortecido com autovalores de malha aberta sobre o eixo imaginário do plano $s$. Em casos como este, pode ser difícil de trabalhar com resposta em frequência, visto que para os traçados temos uma descontinuidade para as frequências coincidentes com os autovalores do sistema sobre o eixo imaginário. O que se encontra na literatura de sistemas de controle segundo a teoria do mapeamento, para contornar esta adversidade, é fazer uma pequena alteração do contorno no plano $s$ de maneira a evitar os polos sobre o eixo $j \omega$, isto pode ser visto como um leve deslocamento para a esquerda dos polos sobre o eixo, que pode ser obtida com uma pequena alteração de um dos elementos da matriz de amortecimento (Ogata 2009). Neste exemplo a matriz D é considerada:

$$
\mathbf{D}=\left[\begin{array}{cc}
1 & -0,9999 \\
-0,9999 & 1
\end{array}\right]
$$

sem qualquer alteração significativa nos valores dos autovalores de malha aberta do sistema.

Novamente a busca pela solução do problema descrito pela equação (3.6) é feita para o círculo $M_{s}=1,667$. Na Tabela 5.3 são exibidos os valores dos vetores de ganho encontrados pela busca por algoritmo genético (método proposto) com e sem restrição adicional e para a alocação de autovalores (proposta em Ram et al. (2009)) com e sem atraso.

\begin{tabular}{|c|c|c|}
\hline Método & $\mathbf{f}$ & $\mathbf{g}$ \\
\hline \hline Alocação com Atraso & {$[-0,0103-0,0103]$} & {$[-0,0213-0,0213]$} \\
\hline \hline Alocação sem Atraso & {$[-2-2]$} & {$[-1-1]$} \\
\hline \hline Método Proposto SRA & {$[-0,0769-0,0833]$} & {$[-0,0526-0,0714]$} \\
\hline \hline Método Proposto CRA & {$[-0,6032-0,0103]$} & {$[-0,2077-0,1111]$} \\
\hline \hline
\end{tabular}

Tabela 5.3: Tabela de Ganhos de Realimentação de Estado - Exemplo 2

Na tabela 5.4 são mostrados os valores de margem de ganho para o sistema do Exemplo 2, com os valores de ganho de realimentação resumidos na tabela 5.3 .

\begin{tabular}{|c|c|}
\hline Método & GM \\
\hline \hline Alocação com Atraso & $27,3 \mathrm{~dB}$ \\
\hline \hline Alocação sem Atraso & $1,57 \mathrm{~dB}$ \\
\hline \hline Método Proposto SRA & $12,7 \mathrm{~dB}$ \\
\hline \hline Método Proposto CRA & $0,769 \mathrm{~dB}$ \\
\hline \hline
\end{tabular}

Tabela 5.4: Margens de ganho - Exemplo 2

O diagrama de Nyquist para o sistema com os ganhos obtidos pela busca pelo método proposto sem restrição adicional é mostrado na Figura 5.6, nele pode-se observar o tangenciamento da curva com a circunferência de raio $\mathrm{M}_{s}^{-1}$ plotada em vermelho. 


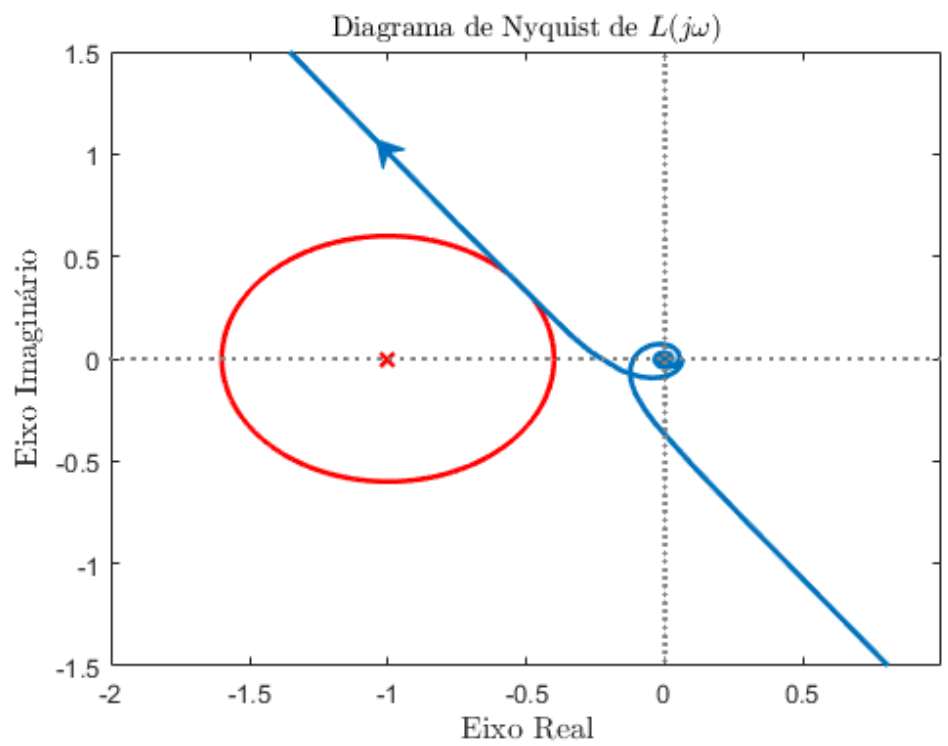

Figura 5.6: Diagrama de Nyquist Exemplo 2 - Método Proposto SRA

Na Figura 5.7 é plotado o diagrama de Nyquist para o sistema com os ganhos obtidos pelo método de alocação de polos com atraso como em Ram et al. (2009); nesse caso a curva de Nyquist de $L(j \omega)$ também se mantém completamente fora da circunferência, porém, muito longe de tangenciar o círculo em qualquer ponto.

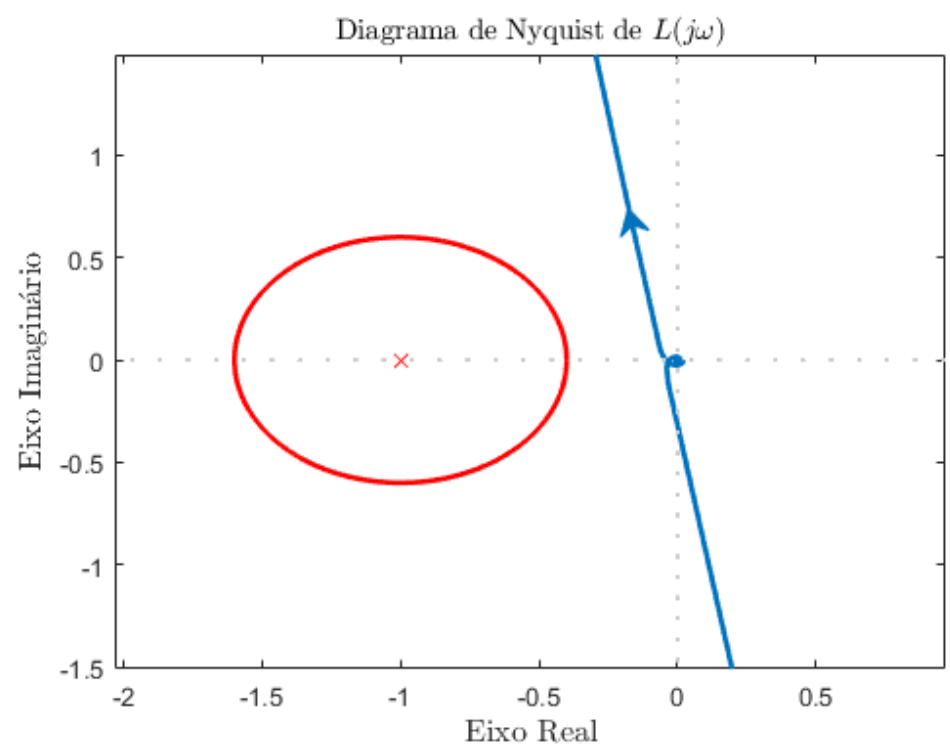

Figura 5.7: Diagrama de Nyquist Exemplo 2 - Alocação com Atraso

O significado desse afastamento do circulo $\mathrm{M}_{s}$ é visto na Figura 5.8, onde são mostradas as respostas do sistema com os ganhos calculados pelo método da alocação com atraso (Figura 5.8a), e pelo método proposto sem considerar a restrição adicional (Figura $5.8 \mathrm{~b}$, para o estado $\mathbf{x}(t)$, sob as condições iniciais $\mathbf{x}(0)=\left[\begin{array}{ll}2 & 0\end{array}\right]^{T}$ e $\dot{\mathbf{x}}(0)=\left[\begin{array}{ll}0 & 0\end{array}\right]^{T}$. O que 
se pode observar da figura é que para o diagrama de Nyquist mais afastado do círculo (Figura 5.7), a resposta temporal é mais lenta.

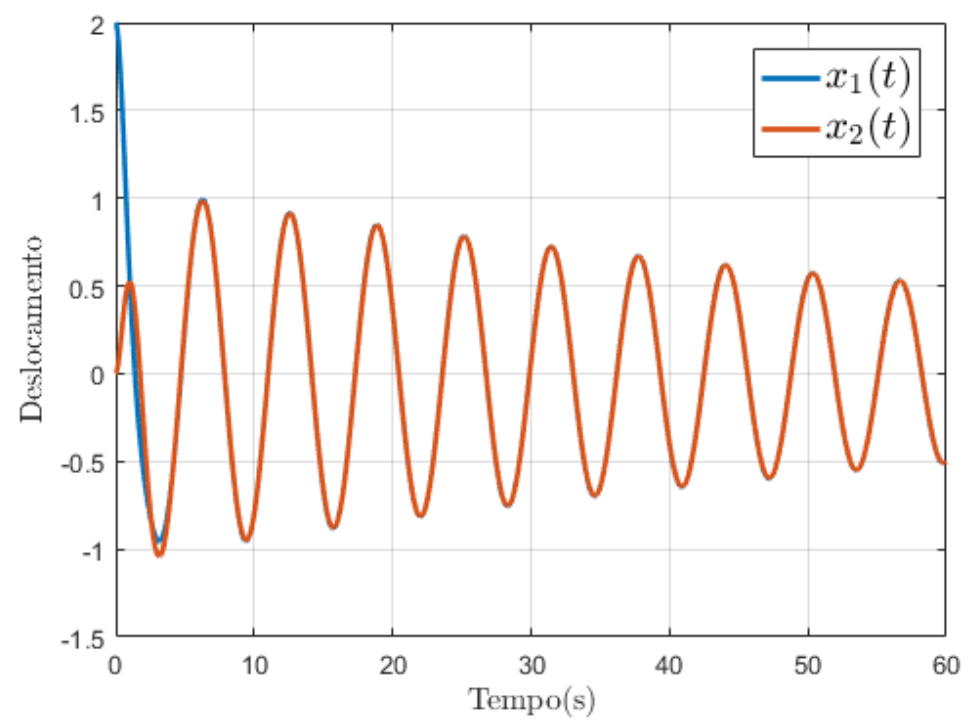

(a) Resposta obtida pelo método de alocação de polos

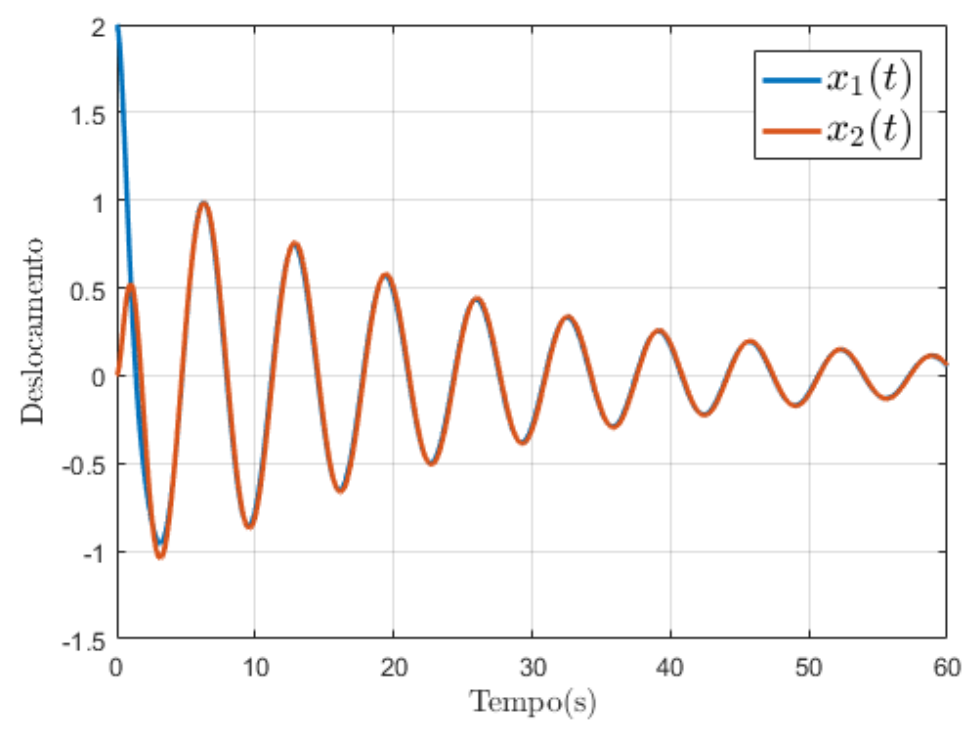

(b) Resposta obtida pelo método proposto SRA

Figura 5.8: Resposta do estado $\mathbf{x}(t)$ para o Exemplo 2

A robustez do sistema associada ao distanciamento da curva de Nyquist do ganho de malha $(L j \omega)$, do ponto $(-1+j 0)$, é corroborada com os valores de margem de ganho da Tabela 5.4. Quanto mais distante do ponto de instabilidade, mais robusto o sistema é. Entretanto, é mostrado na Figura 5.8 que afastar demais a curva de $L(j \omega)$ da circunferência $\mathrm{M}_{s}$ pode influenciar negativamente no desempenho da resposta temporal, por esse motivo a função objetivo do problema de otimização visa tangenciar a circunferência e 
não somente se manter fora dela.

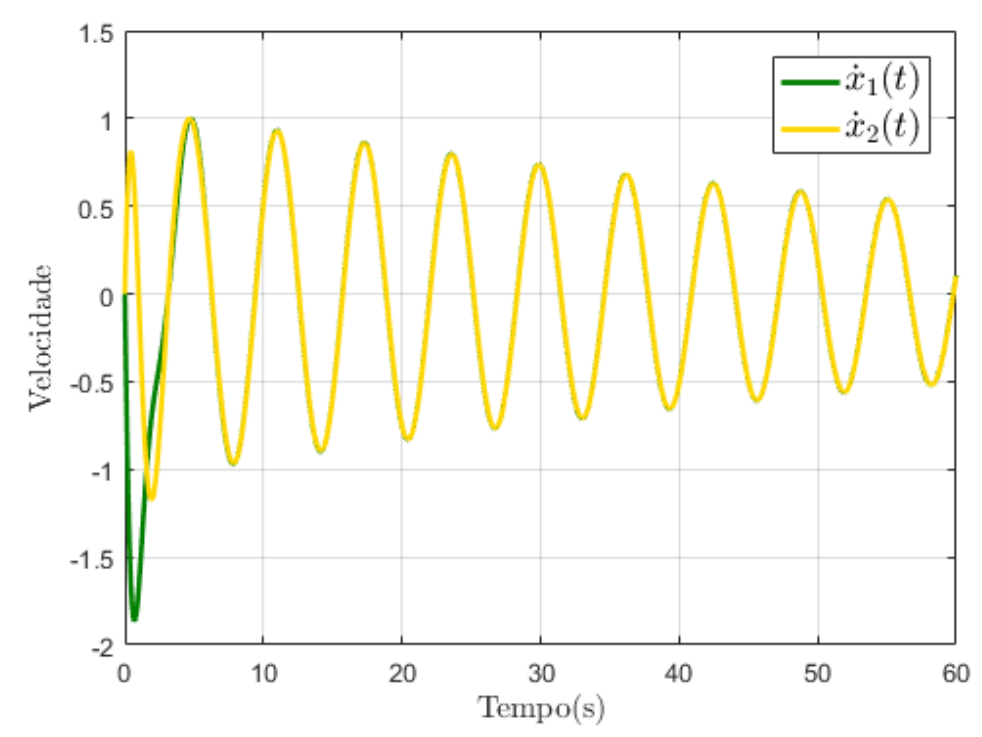

(a) [Resposta obtida pelo método de alocação de polos

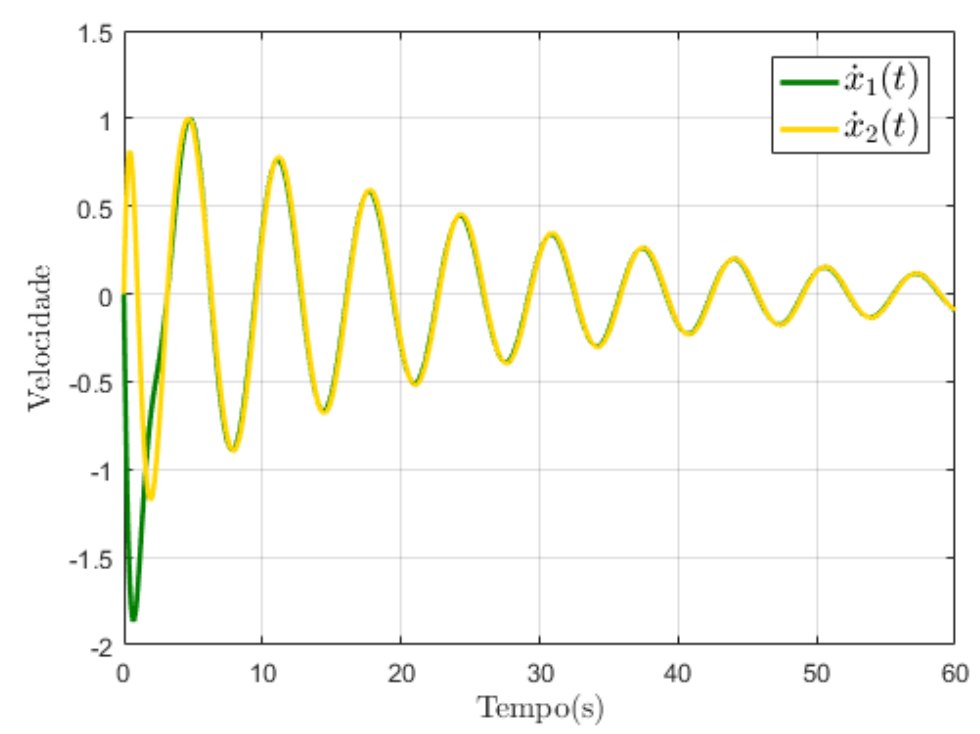

(b) Resposta obtida pelo método proposto SRA

Figura 5.9: Resposta do estado $\dot{\mathbf{x}}(t)$ para o Exemplo 2

Na Figura 5.9 é mostrada a resposta para a derivada do estado, $\dot{\mathbf{x}}(t)$. Aqui novamente cabe o comparativo de desempenho com relação aos resultados para o método de alocação e para o método proposto, sem considerar a restrição adicional, e as conclusões a respeito do afastamento da curva do ganho de malha do sistema do ponto de instabilidade.

Para os ganhos obtidos pelo método da alocação de autovalores, como em Ram et al. (2009), sem levar em consideração o atraso do sistema, o diagrama de Nyquist de $L(j \omega)$ plotado na Figura 5.10 mostra que, segundo o critério de estabilidade de Nyquist, o sis- 


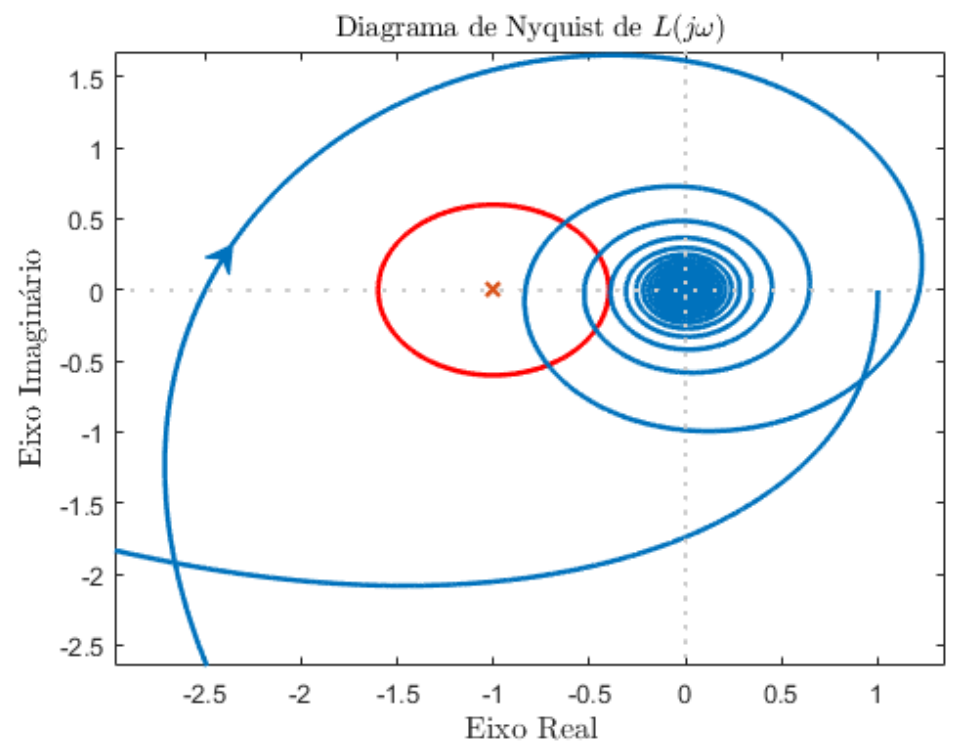

Figura 5.10: Diagrama de Nyquist Exemplo 2 alocação desconsiderando o atraso

tema é instável, dado o envolvimento do ponto $(-1,0)$ pela curva.

Na Figura 5.11 é plotado o diagrama de Nyquist de $L(j \omega)$ para os vetores de ganho obtidos com o método proposto considerando a restrição adicional da Equação (3.9), nela pode-se observar a tangência da curva de Nyquist com a circunferência $\mathbf{M}_{s}$ nas proximidades do eixo real, como desejado.

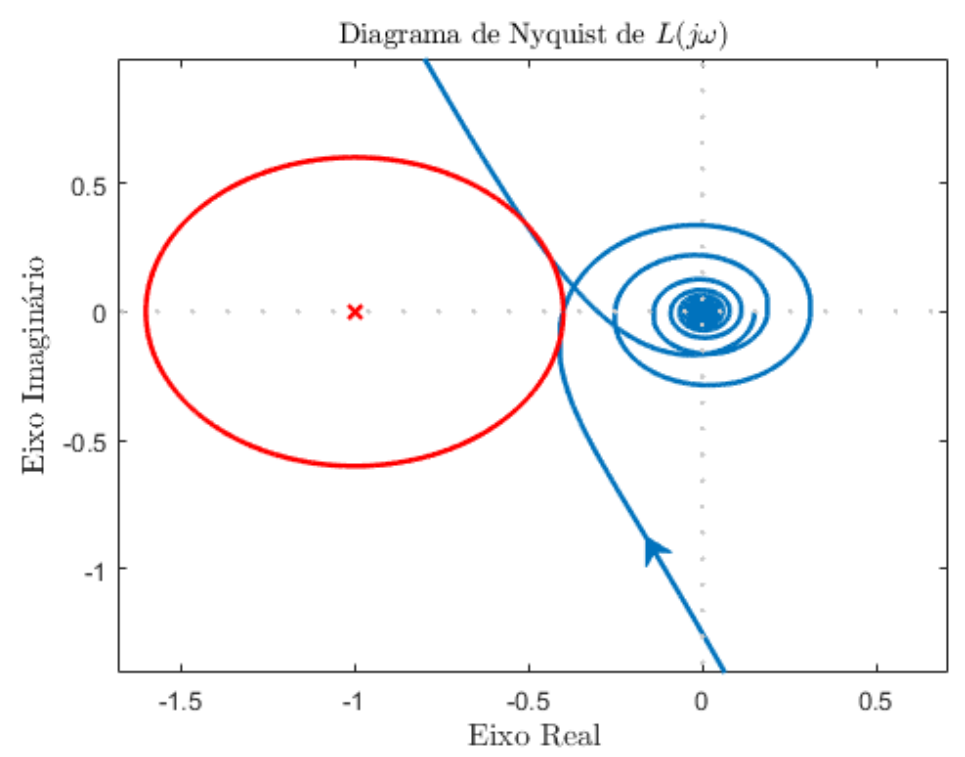

Figura 5.11: Diagrama de Nyquist Exemplo 2 - Controlador com restrição adicional

Para os ganhos obtidos pelo método proposto considerando a restrição adicional, as respostas temporais para o estado $\mathbf{x}(t)$, e para a derivada do estado $\dot{\mathbf{x}}(t)$, são exibidas na Figura 5.12. O que se observa em comparação as respostas ilustradas nas figuras $5.4 \mathrm{e}$ 
5.5 é que para o caso em que se considera a restrição adicional (de tangência no ponto sobre o eixo real) a resposta é consideravelmente mais rápida que as outras, tornando essa parte complementar do método, fundamental na obtenção respostas com melhores desempenhos.

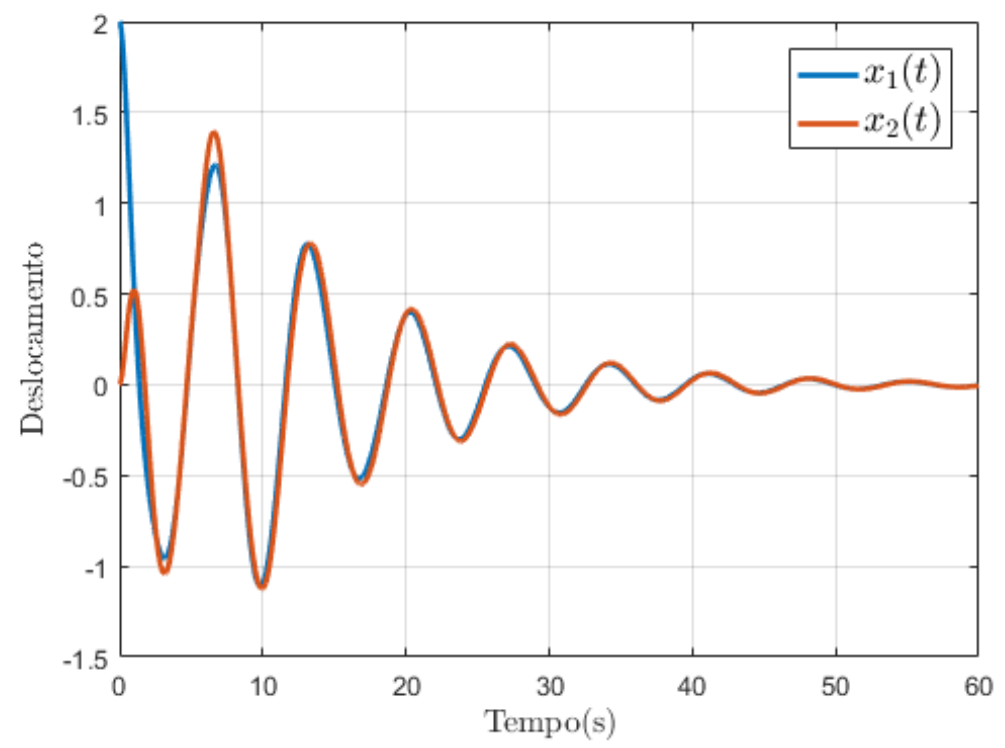

(a) Estado $\mathbf{x}(t)$

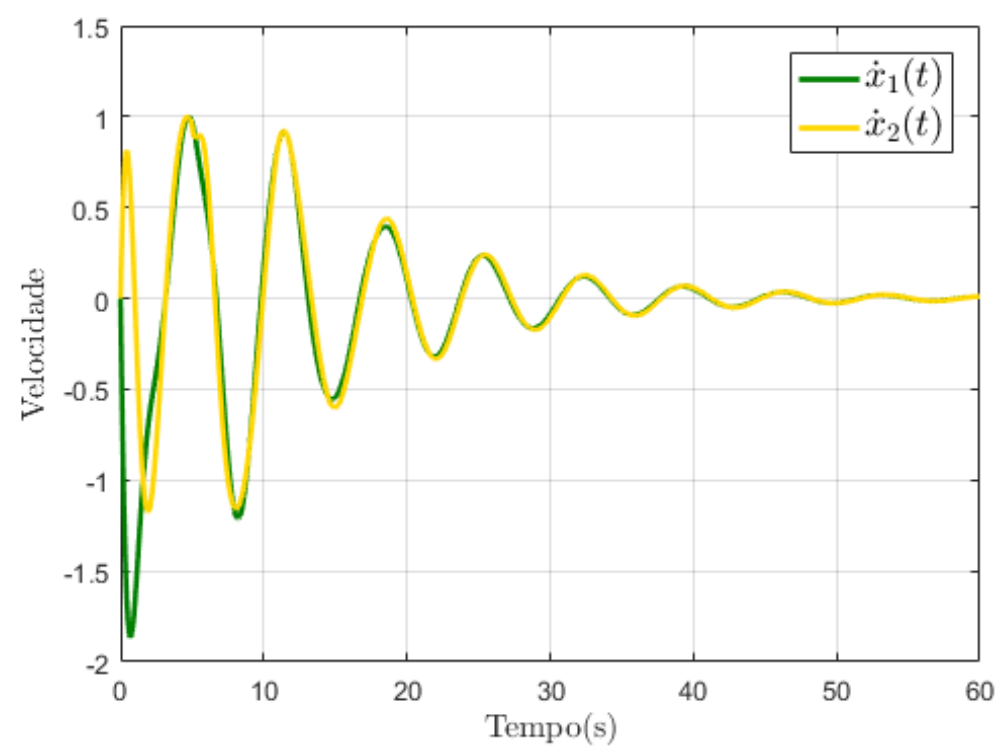

(b) Estado $\dot{\mathbf{x}}(t)$

Figura 5.12: Resposta para os estados $\mathbf{x}(t)$ e $\dot{\mathbf{x}}(t)$ para os ganhos de controlador com restrição adicional 


\subsection{Exemplo 3}

Para o caso de um sistema com três graus de liberdade, emprestado de Singh et al. (2014) com algumas alterações, tendo as matrizes de massa, amortecimento e elasticidade como:

$$
\mathbf{M}=\left[\begin{array}{ccc}
10 & 0 & 0 \\
0 & 10 & 0 \\
0 & 0 & 10
\end{array}\right], \mathbf{D}=\left[\begin{array}{ccc}
5,0 & 0 & 0 \\
0 & 2,5 & 0 \\
0 & 0 & 0,5
\end{array}\right], \mathbf{K}=\left[\begin{array}{ccc}
1500 & -500 & 0 \\
-500 & 600 & -100 \\
0 & -100 & 100
\end{array}\right]
$$

Para uma matriz de entradas $\mathbf{B}^{T}=\left[\begin{array}{lll}1 & 0 & 0\end{array}\right]$ e uma constante de atraso $\tau=0,7$.

Inicialmente para a solução do problema de controle por alocação de autovalores, considera-se o conjunto de autovalores desejados, dados por:

$$
\Lambda^{T}=\left[\begin{array}{lll}
-0,2 \pm j 2,5 & -0,4 \pm j 6,0 \quad-0,6 \pm j 10,0
\end{array}\right]
$$

O método de alocação de autovalores, proposto por Ram et al. (2009), falha como no Exemplo 1, apresentando vetores de ganho que alocam polos primários no semiplano direito. Utilizando a abordagem proposta neste trabalho, para uma circunferência $\mathbf{M}_{s}$ de raio 0,5 , os vetores de ganho encontrados são: $\mathbf{f}=\left[\begin{array}{llll}0,0147 & 6,9622 & 6,4320\end{array}\right] \quad \mathbf{g}=$ $[3,0064-3,99028,8771]$.

O diagrama de Nyquist do ganho de malha $L(j \omega)$ para o sistema deste exemplo com os ganhos $\mathbf{f}$ e $\mathbf{g}$ citados é exibido na Figura 5.13. Para esses vetores de ganho a margem de ganho do sistema é $\mathbf{G M}=6,04 d B$.

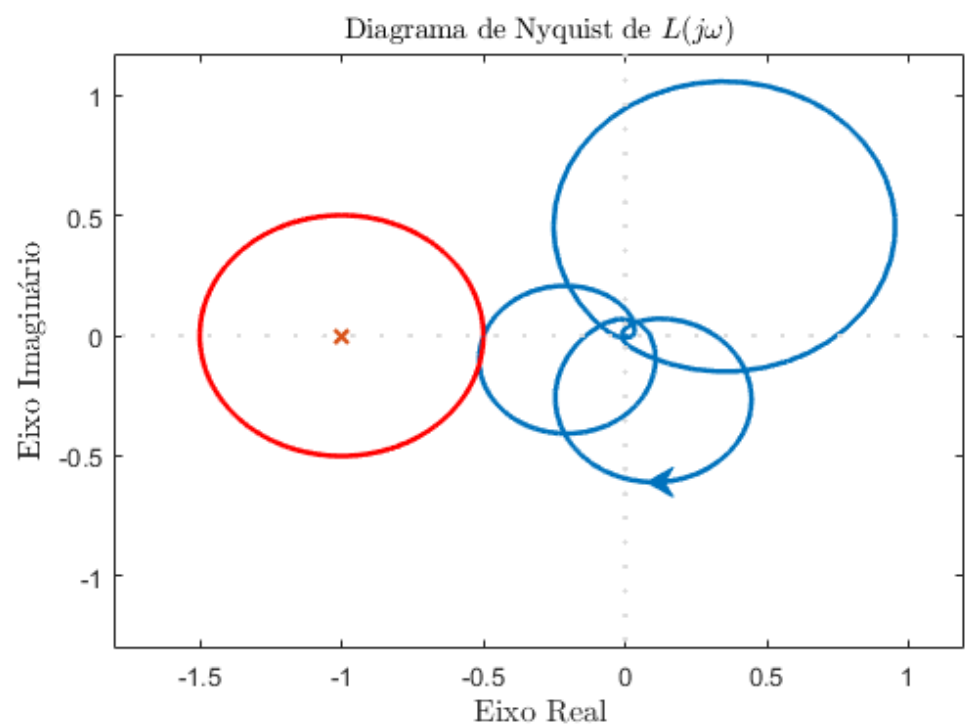

Figura 5.13: Diagrama de Nyquist do Exemplo 3 para os ganhos obtidos pelo método proposto com a restrição adicional

As respostas temporais dos estados, $\mathbf{x}(t)$, e da derivada dos estados, $\dot{\mathbf{x}}(t)$, são exibidas na Figura 5.14 


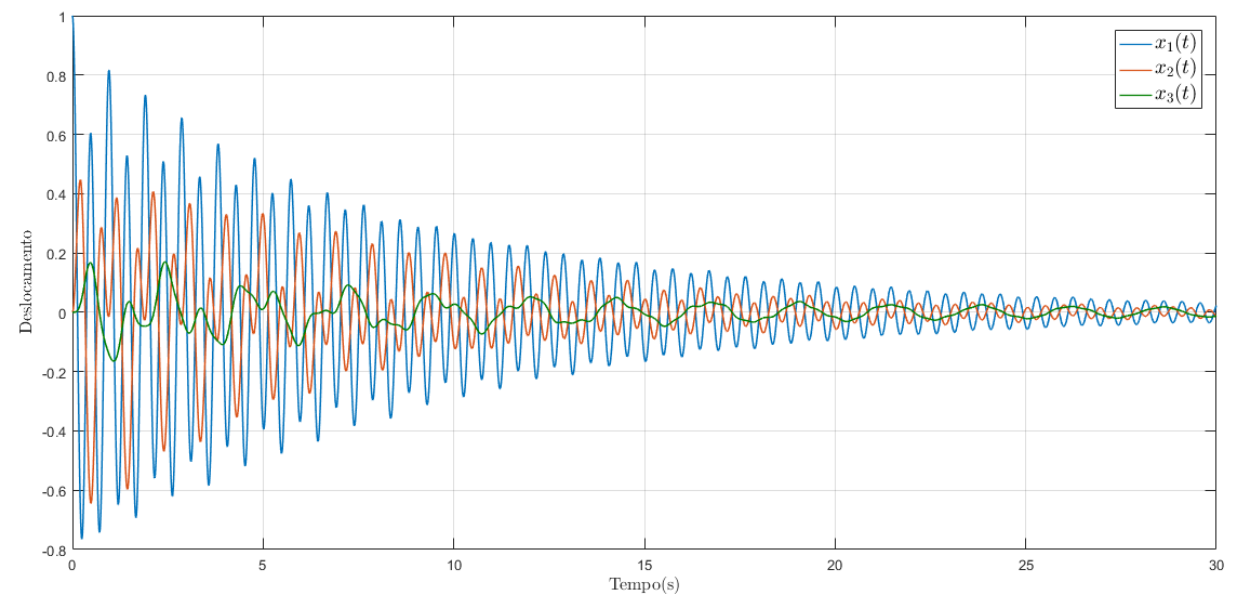

(a) Resposta $\mathbf{x}(t)$

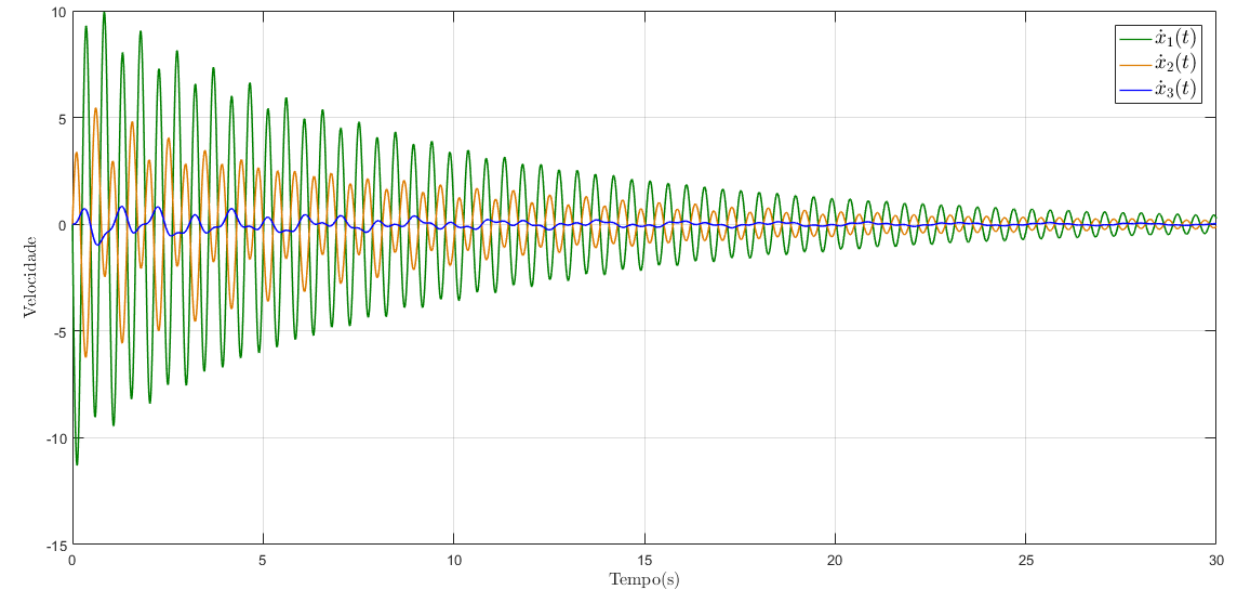

(b) Resposta $\dot{\mathbf{x}}(t)$

Figura 5.14: Resposta Temporal Sistema 3 Graus de Liberdade - Exemplo 3 


\subsection{Exemplo 4}

Utilizando o Exemplo 1 como modelo, realizaram-se buscas para diferentes valores de circunferência $\mathrm{M}_{s}$ para que fossem-se feitas análises sobre o comportamento dinâmico do sistema em diferentes situações para valores variados de raio da circunferência $\mathrm{M}_{s}$. A tabela 5.5 resume os valores de ganho encontrados com a utilização do método de busca proposto para o caso do Exemplo 1.

\begin{tabular}{|r|c|c|}
\hline Raio $\mathbf{M}_{s}^{-1}$ & $\mathbf{f}$ & $\mathbf{g}$ \\
\hline \hline 1,3333 & $-2,9997$ & $-0,4081$ \\
\hline \hline 1,6667 & $-4,4924$ & $-5,8803$ \\
\hline \hline 2,0000 & $-3,2829$ & $-9,9195$ \\
\hline \hline
\end{tabular}

Tabela 5.5: Tabela de Ganhos para Diferentes valores $\mathrm{M}_{s}$

As circunferências $\mathbf{M}_{s}$ listadas na Tabela 5.5 podem ser vistas na Figura 5.15, onde estão plotados os diagramas de Nyquist do sistema apresentado no Exemplo 1, para os respectivos ganhos obtidos pela busca realizada com a aplicação do método proposto, para os diferentes valores de circunferência.

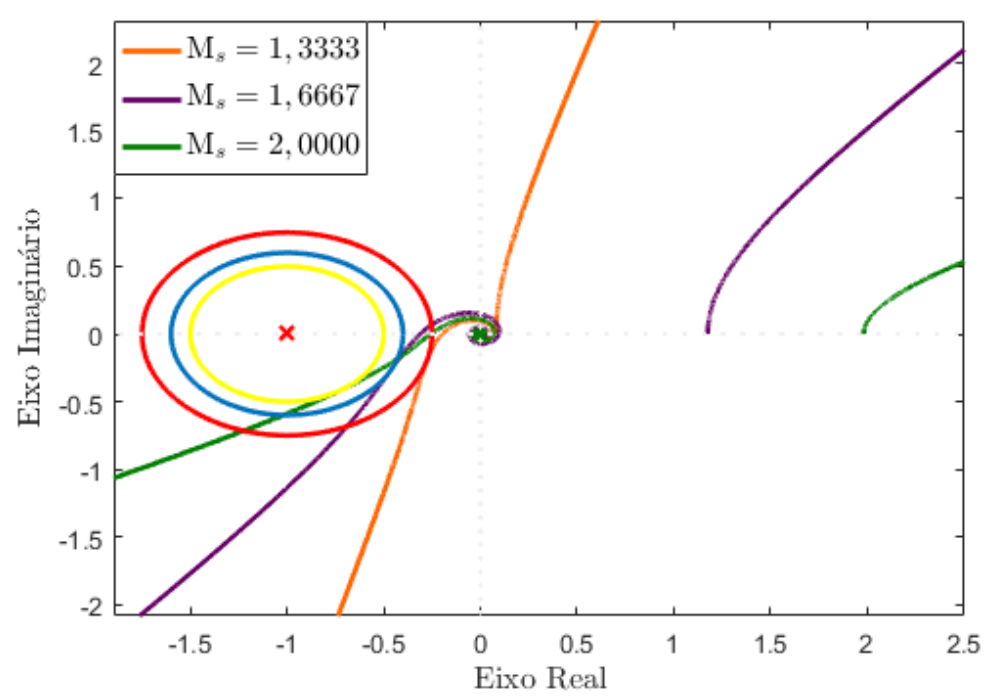

Figura 5.15: Diagrama de Nyquist - Diferentes casos $\mathrm{M}_{s}$

Em comparação com a resposta temporal pode-se observar a coerência com as características de um sistema de segunda ordem típico de um grau de liberdade, onde, por exemplo, a curva correspondente a circunferência de menor raio, que está mais próxima do ponto de instabilidade, apresenta uma resposta no tempo mais oscilatória. 


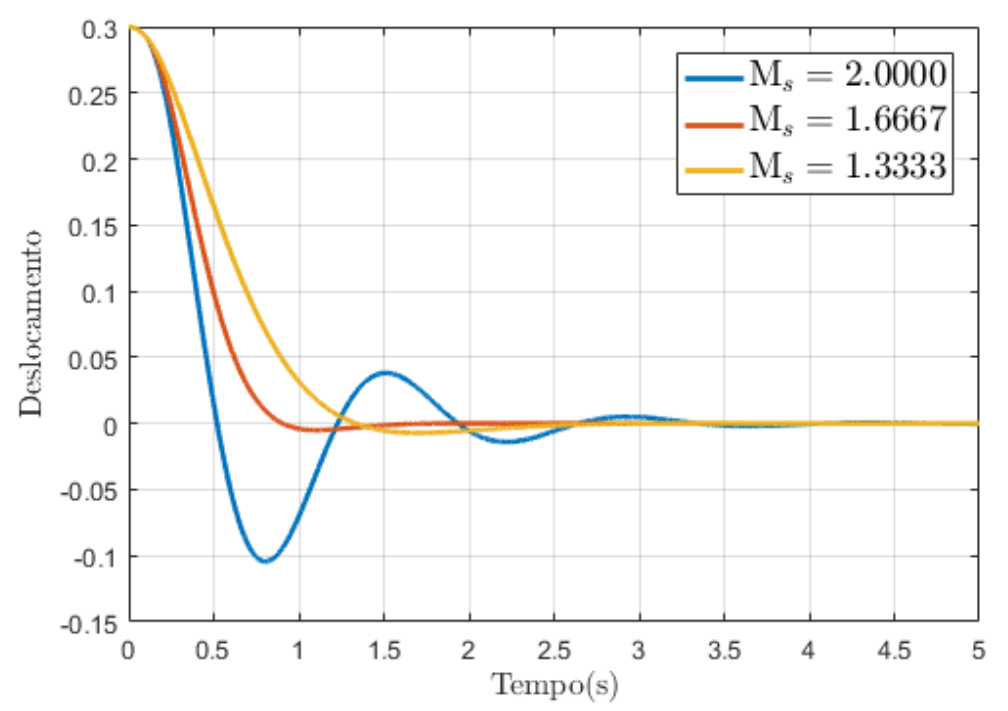

Figura 5.16: Resposta do Estado $x(t)$ - Diferentes casos $\mathrm{M}_{s}$

Por fim, o capítulo 6 aborda os aspectos gerais do trabalho exibindo pontos conclusivos sobre o desenvolvimento e aplicação do método, assim como também sugestões futuras para trabalhos a serem desenvolvidos. 


\section{Capítulo 6}

\section{Conclusão}

Neste trabalho foram apresentadas soluções para o controle de sistemas de segunda ordem com atraso usando abordagem por resposta em frequência, dispensando a necessidade do uso de aproximações e análise a posteriori das soluções encontradas. A utilização da representação dos sistemas de segunda ordem com atraso por matriz de receptância permite que o modelo seja obtido de maneira experimental, o que em casos de sistemas com maior complexidade torna-se uma alternativa muito desejada. O problema de controle foi formulado matematicamente como um problema de otimização para a obtenção dos vetores de ganho do controlador $\mathbf{f}$ e $\mathbf{g}$ e utilizou-se do algoritmo genético como método de busca pela solução. Na aplicação do método com os exemplos numéricos apresentados, em comparação com outros métodos propostos, a resposta obtida mostrou-se satisfatória, alcançando o objetivo de respeitar o critério de projeto definido, de não interceptar o círculo $\mathrm{M}_{s}$, garantindo estabilidade robusta. Para os casos em que se considerou a restrição adicional de tangenciar a curva de Nyquist de $L(j \omega)$ com a circunferência $\mathbf{M}_{s}$, as respostas obtidas foram consideravelmente mais rápidas, apresentando assim, melhor desempenho em relação às outras.

Com a finalização do trabalho apresentado fica como sugestão para trabalhos futuros uma investigação mais aprofundada sobre as relações entre os parâmetros da resposta em frequência utilizadas e a resposta no tempo do sistema, juntamente com buscas feitas para o controle por realimentação derivativa, visto que o equacionamento matemático para os modelos é similar ao obtido para o caso de realimentação de estados, além de análises de possíveis modificações no método de busca, visando aprimorar o desempenho. 


\section{Referências Bibliográficas}

Araújo, José Mário (2018a), 'Discussion on 'state feedback control with time delay", Mechanical Systems and Signal Processing 98, 368-370.

Araújo, José Mário (2018b), 'Partial eigenvalue assignment inlinear time-invariant systems using state-derivative feedback and a left eigenvectors parametrization', Proceedings of the Institution of Mechanical Engineers, Part I: Journal of Systems and Control Engineering p. 0959651818811010.

Araújo, José Mário \& Tito Luís Maia Santos (2018), 'Control of a class of second-order linear vibrating systems with time-delay: Smith predictor approach', Mechanical Systems and Signal Processing 108, 173-187.

Balas, Mark (1982), 'Trends in large space structure control theory: fondest hopes, wildest dreams', IEEE Transactions on Automatic Control 27(3), 522-535.

Chu, EK \& BN Datta (1996), 'Numerically robust pole assignment for second-order systems', International Journal of Control 64(6), 1113-1127.

Datta, Biswa (2004), Numerical methods for linear control systems, Vol. 1, Academic Press.

Datta, Biswa N, Sylvan Elhay \& Yitshak M Ram (1997), 'Orthogonality and partial pole assignment for the symmetric definite quadratic pencil', Linear algebra and its applications 257, 29-48.

Euzébio, Thiago AM \& Péricles R Barros (2015), 'Iterative procedure for tuning decentralized pid controllers', IFAC-PapersOnLine 48(8), 1180-1185.

Euzébio, Thiago Antonio Melo \& Péricles Rezende Barros (2013), 'Optimal integral gain for smooth pi control', IFAC Proceedings Volumes 46(11), 529-533.

Franklin, Gene F, J David Powell \& Abbas Emami-Naeini (2013), Sistemas de controle para engenharia, Bookman Editora.

Goldberg, David E \& John H Holland (1988), 'Genetic algorithms and machine learning', Machine learning 3(2), 95-99.

Golub, Gene H \& Charles F Van Loan (2012), Matrix computations, Vol. 3, JHU Press.

Juang, Jer-Nan \& Peiman G Maghami (1990), Robust eigensystem assignment for secondorder dynamic systems, American Institute of Aeronautics and Astronautics. 
Liu, Hao \& Yongxin Yuan (2016), 'A multi-step method for partial quadratic pole assignment problem with time delay', Applied Mathematics and computation 283, 29-35.

MathWorks (2019), 'Genetic algorithm - MATLAB \& Simulink'.

URL: https://www.mathworks.com/help/gads/genetic-algorithm.html

Mottershead, John E \& Yitshak M Ram (2006), 'Inverse eigenvalue problems in vibration absorption: passive modification and active control', Mechanical systems and signal processing 20(1), 5-44.

Mottershead, John E \& Yitshak M Ram (2007), 'Receptance method in active vibration control', AIAA journal 45(3), 562-567.

Ogata, Katsuhiko (2009), Modern control engineering, Prentice Hall Upper Saddle River, NJ.

Ram, YM, Akshay Singh \& John E Mottershead (2009), 'State feedback control with time delay', Mechanical Systems and Signal Processing 23(6), 1940-1945.

Ram, YM, JE Mottershead \& M Ghandchi Tehrani (2011), 'Partial pole placement with time delay in structures using the receptance and the system matrices', Linear Algebra and its Applications 434(7), 1689-1696.

Santos, Tito LM, José Mário Araújo \& Taniel S Franklin (2018), 'Receptance-based stability criterion for second-order linear systems with time-varying delay', Mechanical Systems and Signal Processing 110, 428-441.

Singh, Kumar V, Rajeeb Dey \& Biswa N Datta (2014), 'Partial eigenvalue assignment and its stability in a time delayed system', Mechanical Systems and Signal Processing 42(1-2), 247-257.

Skogestad, Sigurd \& Ian Postlethwaite (2007), Multivariable feedback control: analysis and design, Vol. 2, Wiley New York.

Van Loan, Charles F \& Gene H Golub (1983), Matrix computations, Johns Hopkins University Press.

Vandervelde, Wallace E (1986), 'Control of large flexible space structures'.

Wonham, W (1967), 'On pole assignment in multi-input controllable linear systems', IEEE transactions on automatic control 12(6), 660-665.

Zhang, Jiafan, Huajiang Ouyang \& Jun Yang (2014), 'Partial eigenstructure assignment for undamped vibration systems using acceleration and displacement feedback', Journal of Sound and Vibration 333(1), 1-12. 